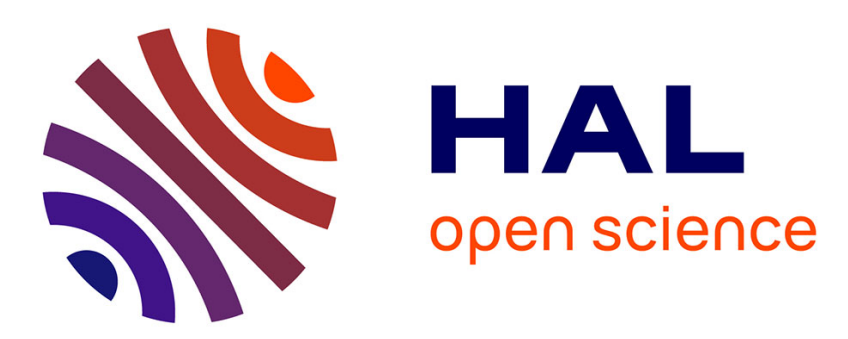

\title{
Modeling the rheological behavior of waxy crude oils as a function of flow and temperature history
}

\author{
Rafael Mendes, Guillaume Vinay, Guillaume Ovarlez, Philippe Coussot
}

\section{To cite this version:}

Rafael Mendes, Guillaume Vinay, Guillaume Ovarlez, Philippe Coussot. Modeling the rheological behavior of waxy crude oils as a function of flow and temperature history. Nihon Reorojī Gakkaishi $=$ Journal of the Society of Rheology, Japan, 2015, 59 (3), pp.703-732. 10.1122/1.4916531 . hal01174631

\section{HAL Id: hal-01174631 \\ https://hal.science/hal-01174631}

Submitted on 9 Jul 2015

HAL is a multi-disciplinary open access archive for the deposit and dissemination of scientific research documents, whether they are published or not. The documents may come from teaching and research institutions in France or abroad, or from public or private research centers.
L'archive ouverte pluridisciplinaire HAL, est destinée au dépôt et à la diffusion de documents scientifiques de niveau recherche, publiés ou non, émanant des établissements d'enseignement et de recherche français ou étrangers, des laboratoires publics ou privés. 


\title{
Modeling the rheological behavior of waxy crude oils as a function of flow and temperature history
}

\author{
Rafael Mendes ${ }^{\text {a) }}$ \\ Petrobras Research Center, Rio de Janeiro, Brazil \\ Guillaume Vinay \\ IFP Energies nouvelles, Rueil-Malmaison, France \\ Guillaume Ovarlez \\ CNRS, LOF, UMR 5258, Pessac, France \\ Philippe Coussot \\ Laboratoire Navier (ENPC-IFSTTAR-CNRS), Université Paris-Est, \\ Champs-sur-Marne, France
}

(Received 11 December 2014; final revision received 12 March 2015;

published 7 April 2015)

\begin{abstract}
Synopsis
The solidification of waxy components during the cool down of waxy crude oils in pipelines may provide complex yield stress fluid behavior with time-dependent characteristics, which has a critical impact for predicting flow restart after pipeline shut-in. Here, from a previous set of data at a local scale with the help of Magnetic Resonance Imaging and a new full set of data for various flow and temperature histories, we give a general picture of the rheological behavior of waxy crude oils. The tests include start flow tests at different velocities or creep tests at different stress levels, abrupt changes of velocity level, steady flow, after cooling under static or flowing conditions. We show that when the fluid has been cooled at rest it forms a structure that irreversibly collapses during the startup flow. Under these conditions, the evolution of the apparent viscosity mainly depends on the deformation undergone by the fluid for low or moderate deformation and starts to significantly depend on the shear rate for larger values. Even the (apparent) flow curve of statically cooled waxy crude oils was observed to be dependent on the flow history, more specifically on the maximum shear rate experienced by the material. After being sufficiently sheared, i.e., achieving an equilibrium state, the rheological behavior is that of a simple liquid for shear rates lower than the maximum historical one. A model is proposed to represent those trends experimentally observed. In contrast with most previous works in that field, the model is built without any a priori assumption based on classical behavior of a class of fluids. Finally, it is shown that this model
\end{abstract}

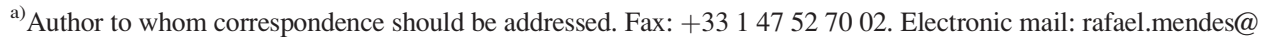
petrobras.com.br
} 
predicts the flow characteristics of these materials under more complex flow histories (sweep tests, sudden shear rate decrease) much better than the so far most often used (Houska) model. (C) 2015 The Society of Rheology. [http://dx.doi.org/10.1122/1.4916531]

\section{INTRODUCTION}

Many crude oils contain dissolved waxes that can precipitate when the temperature falls below the WAT (wax appearance temperature). Crystals start forming and the rheological behavior of the crude oil turns from a simple Newtonian to a much more complex behavior and can now exhibit a high yield stress and time-dependent characteristics. Thus, the transport of waxy crude oils through long pipelines in cold environments involves handling some huge flow assurance issues, such as the management of wax deposition at the pipe walls or high pressure gradients needed for flow restart after stoppage, with the possibility of additional issues, e.g., appearance of void fractions during cool down at rest [Hénaut et al. (1999); Philips et al. (2011a)] and pressure propagation effects [Philips et al. (2011b); El-gendy (2012)].

The solidification process in a waxy crude oil under cooling is a complex process due to the different paraffin molecules and hydrocarbon matrix interactions [Rønningsen et al. (1991); Kané et al. (2003)]. As the crystals grow they may form aggregates eventually forming a volume-spanning network which may be seen as a colloidal-type gel [Lopes-da-Silva and Coutinho (2007)]. Due to the complexity of the system, the characteristics of this structure obviously depend on the timing of cooling but also on the fluid motion during cooling, which induces relative displacements of species and possible aggregate breakage. The appearance of solid deposits of paraffins at the pipe wall is an additional phenomena that may disturb the flow in the long term. However, noticeable composition changes in the flowing oil are not expected in production flowlines. Moreover, even at the end of cooling and phase change, the oil-gel may still be out of equilibrium, allowing further isothermal rearrangements [Lopes-da-Silva and Coutinho (2007); Jia and Zhang (2012)]. Therefore, the resulting rheological behavior of the gel is a function not only of the oil composition but also of the cooling rate and the flow history during all the cooling process [Rønningsen (1992); Ekweribe et al. (2008)]. This has a critical impact for predicting pipe flow restart since the fluid at the pipe entrance and the fluid far downstream from the entrance have generally undergone very different flow histories and cooling processes.

Several works in the literature were devoted to characterize waxy oils yield stress after different cooling histories. Typically, lower cooling rates, lower shear rates, and longer holding times at rest give rise to higher yield stresses, when independently analyzed [Venkatesan et al. (2005); Lin et al. (2011); Zhao et al. (2012)]. The oil-gel shear stress response at low deformations, with the objective of analyzing its detailed yielding characteristics, were also studied by Chang and Boger (1998), Kané et al. (2004), Magda et al. (2009), and Oh et al. (2009), for example.

However, few studies describe the flow evolution of waxy crude oils beyond yielding and its representation by pertinent rheological models. In literature, this has been done mostly through modeling. A work of reference in that field is that of Houska (1981), then used by Sestak et al. (1987), Cawkwell and Charles (1989), and Wachs et al. (2009), who described the rheological behavior by a model in the range of classical thixotropic models for colloidal dispersions [see Coussot (2005) and Mewis and Wagner (2009)]. That model is the result of an evolution of representing thixotropic flows by generalizing the Bingham model. It includes a first equation of the Herschel-Bulkley (HB) type with yield 
stress and viscous coefficient depending on a structure parameter, and a second (kinetic) equation describing the evolution of the structure parameter as a function of flow history, at constant temperature. In this description, the yield stress can vary from a low value, corresponding to a fully destructured fluid, to a high value, for a fully structured material. In that case, the parameter determination is done by rheometry, exploring different shear values and structure parameter evolutions [see Cawkwell and Charles (1989), Hénaut and Brucy (2001), and Wang and Huang (2014)], sometimes with a new version of the model involving more parameters [Ding et al. (2006)]. However, in the literature, one can hardly find a discussion of the quality of this model to describe the effective rheological behavior of such materials obtained from careful and systematic rheometrical data.

Some recent works provided more sophisticated modeling approaches of the behavior of thixotropic materials, relying on interesting physical arguments. De Souza Mendes (2011) used a shear stress equation with the form of a linear viscoelastic Maxwell constitutive equation, but with variable parameters that depend on a structural parameter. The kinetic equation for the structure parameter includes a characteristic relaxation time and the shear stress is the driving force for the structure breakage. On another side, Teng and Zhang (2013) represent the material stress response by adding an elastic and a viscous tress term. In the elastic stress, the shear modulus is assumed to vary proportionally to a structural parameter and a nonlinear damping function. The viscous stress term is proportional to a structure-dependent consistency parameter and a completely unstructured one, as in the Houska model.

The kinetic equation for the structure parameter assumed a characteristic time that depends on the structure parameter itself and the driving force for the structure breakage is the flow energy dissipation. The predictions of this model were successfully compared with experimental data for start flow after cooling at rest, but only in the destructuring stage. At last, Dimitriou and McKinley (2014) developed a rheological constitutive equation that captures the main features observed in LAOS (large amplitude oscillatory shear) experiments with a model waxy oil. In that work, the material deformation was modeled in two separated components: A linear viscoelastic and a plastic deformation. The concept of isotropic-kinematic hardening is applied by allowing a plastic deformation only when the stress is higher than a yield stress added to a back stress, which tracks the location of the center of the material yield surface with the stress direction. The elastic part of the deformation is modeled with constant parameters, thus carrying the strong assumption (see below) that $\mathrm{G}^{\prime}$ and $\mathrm{G}^{\prime \prime}$ do not vary with changes in the material structure. Their analysis was focused on the physical behavior of the model waxy oil in its slurry state, i.e., before the tests, the sample was highly sheared at the test temperature, erasing stronger gel characteristics that would be obtained from static cooling, for example. By doing so, they could model and predict thixotropic phenomena around the flow curve of the material, which were called reversible structure changes by Mendes et al. (2014). Experimentally measured stress overshoots and a negative slope in a local shear stress vs local shear rate were well predicted by the model.

Actually, in view of describing the flow characteristics during startup, there is a strong issue with the above descriptions. They implicitly assume that the material exhibits reversible rheological properties, which somewhat restricts the range of evolution of the structure parameter. Hence, it does not allow to fit the model to experimental data obtained after different cooling histories, which in particular, may show a strong decrease of the apparent viscosity. As a consequence, another class of work focused on the behavior evolution during start up as a function of cooling and flow histories. Visintin et al. (2005), besides evaluating the oil-gel restructuration by following its $\mathrm{G}^{\prime}$ behavior and viscosity evolution after shear rate step changes, used a time-dependent viscosity model 
with two asymptotic viscosities (zero-shear rate and infinite-shear rate viscosities) for describing its experimental data on destructuring flow. Roningsen (1992) found that a time-dependent Bingham model with constant plastic viscosity could be another approximation to represent the viscosity decay of the studied fluids. Later, Chang et al. (1999) applied that model in pipeline flow restart calculations, representing the time variations of the yield stress term in the above model. Aiming for a different approach, Kané et al. (2004) evaluated the apparent viscosity behavior with time by imposing shear rate steps, from 10 to $500 \mathrm{~s}^{-1}$, after dynamic cooling at $10 \mathrm{~s}^{-1}$. They related those data with oil-gel structural observations of a previous work [Kané et al. (2003)] in a fractal description of the media. They concluded, however, that it was not entirely possible to relate the phenomenological parameters of the model for flow description and the structural features of the gel networks.

The fact that so far either the behavior within the frame of the usual thixotropic context (possibly with some sophistications for describing the solid-liquid transition) or the strong viscosity variations during start up after different cooling histories have been specifically considered clearly means that further work is needed to encompass both aspects.

A simple test (see Fig. 1) illustrates these complex rheological characteristics of waxy crude oil statically cooled to a temperature below the WAT. We submit the material to a shear rate ramp up and down. We have first a rapid increase of the stress with the shear rate, as for a yield stress fluid in the solid regime, then beyond a maximum value which likely corresponds to the solid-liquid transition the stress falls despite the increasing shear rate, indicating an important destructuring process. With further shear rate increase the stress finally increases again, forming a nonmonotonic transient shear stress vs shear rate curve during this flow start. Finally when the shear rate is decreased, the stress is significantly below the ramp-up curve.

At first sight, the trends observed in this test suggest that the waxy crude oil exhibits a yield stress and a common thixotropic behavior, due to the important hysteresis in the upand-down curves. In this frame, the local minimum in the stress vs shear rate curve, which cannot correspond to homogeneous steady flows [Tanner (2000); Coussot (2005)],

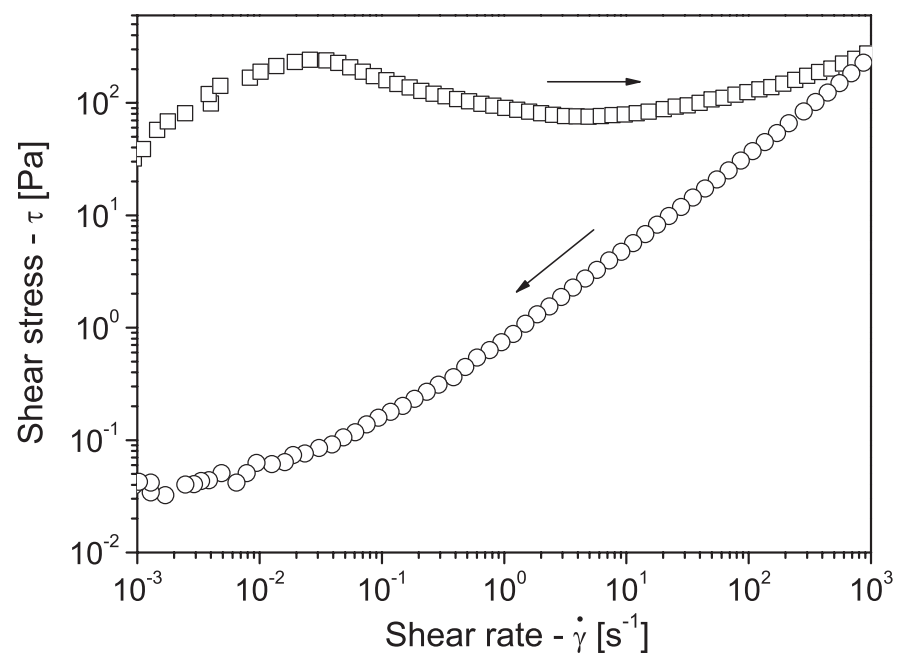

FIG. 1. Shear stress vs shear rate during a sweep test with a waxy crude oil after static cooling from $60^{\circ} \mathrm{C}$ to $4{ }^{\circ} \mathrm{C}$ at $-1{ }^{\circ} \mathrm{C} / \mathrm{min}$ and holding at $4{ }^{\circ} \mathrm{C}$ for $20 \mathrm{~min}$. The up and down ramps were performed in 5 min each with a logarithmic variation [source: Mendes et al. (2014)]. 
is consistent with observations for strongly thixotropic fluids [Coussot et al. (1993); Pignon et al. (1996); Møller et al. (2008)].

However, the results of Fig. 1 also show intriguing trends:

- The decreasing curve reveals a linear fluid behavior in a (arbitrary) limited range of shear rates with negligible yield stress with respect to the increasing curve;

- It strongly departs from the increasing curve since the stress level is situated three orders of magnitude below;

- With such a difference between the two curves, the question is open as to whether the material behavior is reversible, i.e., can we get back the initial yield stress after leaving the fluid at rest for a sufficient time?

The recent study of Mendes et al. (2014) using magnetic resonance rheometry provided some explanations to these observations and showed that the thixotropic characteristics observed in waxy crude oils differ from the classical concept. The fluid steady state flow curve critically depends on its flow history at the temperature achieved after the cooling process. Each time the waxy oil is submitted to a shear rate higher than it has ever seen, an irreversible degradation of the fluid rheological parameters takes place, redefining its steady state flow curve.

In the present work, we intend to clarify these different aspects, namely, the relation between the start flow characteristics after different cooling and flow histories and the subsequent rheological behavior (possibly thixotropic), and the question of the reversibility of the destructuring process during this start flow. In that aim we will carry out a complete rheometrical analysis of two waxy crude oils including start flow tests at different velocities, abrupt changes of velocity level, steady flow, sweep tests, for different cooling and flow histories. In parallel, we will set up a model describing the different trends observed at each step of the rheological study. In contrast with most previous works in this field, this modeling approach will thus be carried out without any a priori assumption concerning the form of the constitutive equation.

\section{MATERIALS AND METHODS}

Two waxy crude oils were analyzed. Crude oil A has density $27^{\circ}$ API (American Petroleum Institute gravity) and WAT of $22^{\circ} \mathrm{C}$. Crude oil B has density $31^{\circ}$ API and WAT of $30^{\circ} \mathrm{C}$. The WAT was measured with a differential scanning calorimeter Mettler Toledo DSC1 Of course, it is not expected here that those two crude oils would represent all possible waxy crude oil rheological behaviors, since each crude oil may have a particular characteristic. As it is not possible to define a representative waxy crude oil in a global sense, though, this work goes beyond the analysis of model waxy oils by investigating two crude oils, which show rheological properties differing of one order of magnitude (see Figs. 12 and 13).

Both oils were sampled from larger containers after mixing and stored in small flasks. No solid deposits were observed at any time in the containers. Before being collected for the rheometry tests, the flasks were heated to a temperature above the WAT. Once loaded in the rheometer, the oil was heated to $60^{\circ} \mathrm{C}$ and sheared for erasing any thermal memory before the beginning of the experiment and then cooled down to $4{ }^{\circ} \mathrm{C}$. When static cooling is employed, the sample is cooled at $-1{ }^{\circ} \mathrm{C} / \mathrm{min}$ and let at rest for $20 \mathrm{~min}$ (holding time) at $4{ }^{\circ} \mathrm{C}$. For cooling under shear, the sample was sheared at $0.1 \mathrm{~s}^{-1}$, without any rest time after that.

Rheometry tests were performed with a TA Instruments AR2000, a controlled stress rheometer, equipped with a Peltier plate, and a $40 \mathrm{~mm}$ diameter serrated plate geometry. 
A trap sitting on the Peltier plate covers the geometry and sample leaving a small opening to the ambient. The gap between the parallel plates was set to $0.6 \mathrm{~mm}$ for crude oil A and $1.0 \mathrm{~mm}$ for oil B. A smaller gap for crude oil A made the gap filling easier. Such gaps are thought to be in the appropriate range according to recommendations of Marchesini et al. (2012), allowing to minimize shrinkage effects [as the conclusions of Hénaut et al. (1999) suggest] and optimize temperature control. Successive similar rheometrical tests carried out with the sample loaded in the geometry and following the same temperature and flow history from the setup were observed to give slightly different results (typically a variation of a few percent). This effect is likely due to evaporation of light ends components. An excellent reproducibility of data was nevertheless observed when the sample loaded in the rheometer was changed after each thermal cycle. After each temperature cycle, the rheometer was cleaned and a new sample was taken from the flasks and loaded into the rheometer.

\section{RESULTS}

Here, we show data obtained at $4{ }^{\circ} \mathrm{C}$ for crude oil A, but qualitatively similar behavior was observed with both oils at different temperatures in the range from 4 to $16^{\circ} \mathrm{C}$ (see Sec. V).

\section{A. Creep tests}

We start with the basic test [see Coussot (2005)] that allows evaluating the yielding characteristics of the fluid, namely, a series of creep tests under different imposed shear stresses. If the stress is below a minimum yield stress limit, after an initial deformation, no flow should be observed. If the applied shear stress is higher than that critical stress, the oil sample should start flowing within some time, depending on the applied stress magnitude.

A set of tests were performed after cooling the oil sample A at rest from $60^{\circ} \mathrm{C}$ to $4{ }^{\circ} \mathrm{C}$ at $-1{ }^{\circ} \mathrm{C} / \mathrm{min}$ and holding time of $20 \mathrm{~min}$. Next, a constant shear stress is applied and the shear rate is measured with time. After an initial deformation, the shear rate corresponding to a stress of $150 \mathrm{~Pa}$ apparently tends to zero, indicating that this stress value is too low to start the flow (see Fig. 2). On the contrary, for stresses equal or larger than $200 \mathrm{~Pa}$, we can observe a dramatic increase of the shear rate which reaches a value of the order of $10^{3} \mathrm{~s}^{-1}$ in a very short time. The shear rate in steady state does not vary much around this value for a stress between 200 and $250 \mathrm{~Pa}$.

Qualitatively, such behavior, i.e., no flow below a critical stress and rapid flow beyond that stress value, is similar to the viscosity bifurcation effect observed for colloidal dispersions [see Coussot et al. (2002)]. However, here the effect is stronger: The shear rate reached for a stress just above the yield stress (here situated between 150 and $200 \mathrm{~Pa}$ ) is very high (several hundreds of $\mathrm{s}^{-1}$ ). Note that this behavior is consistent with the observations of Fig. 1: The flow starts for a stress in the order of $200 \mathrm{~Pa}$ and the shear rate rapidly reached a high value since no stable flows at lower shear rates can be obtained under the given stress.

It is worth noting that for the different stress values the abrupt increase of the shear rate, associated with the transition to the liquid regime, occurs for the same critical deformation (around 0.15), as the corresponding deformation vs time curves show in the inset in Fig. 2. That means that the material first undergoes a creep flow in its solid regime and falls in the liquid regime when it has reached its critical deformation. Here, we consider that at the solid-liquid transition, yielding affects the initial solid structure configuration 


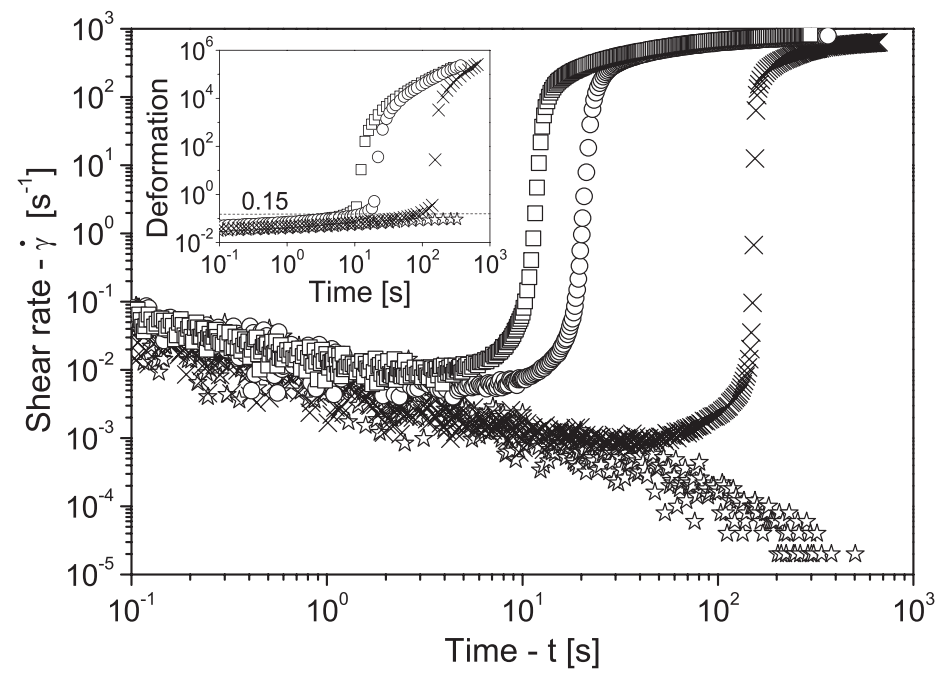

FIG. 2. Shear rate vs time for different constant stresses [150 (仿—stars), 200 ( $\times$ —crosses), 225 ( $\bigcirc —$ circles), and $250 \mathrm{~Pa}$ ( $\square-$ squares)] applied to crude oil A cooled at rest. The inset shows the equivalent deformations.

by irreversibly breaking it. Then, in the liquid state plastic effects take place [see Coussot (2005)]. The shear rate in the creep flow decreases when the stress is decreased and approaches the yield stress. It means that the time before reaching the liquid regime will increase at the approach of the yield stress, which complicates the exact determination of the yield stress value in practice. This constitutes an additional difficulty for studying the flow properties of these materials, explaining why, in the following, we will focus on controlled shear rate tests.

\section{B. Constant shear rate tests}

Fluids manifesting viscosity bifurcation effect also exhibit shear banding in steady state when a shear rate is imposed below a critical value reached at a stress just above the yield stress [Ovarlez et al. (2009)]. In that case, the thickness of the shear localization band decreases with the apparent shear rate value and the shear rate along the interface between the solid and the liquid regions is finite and can even be constant if the stress distribution is homogenous. This effect results from the competition between destructuring and restructuring processes in these thixotropic fluids [Coussot et al. (2002)].

The recent MRI (Magnetic Resonance Imaging) rheometry study [Mendes et al. (2014)] of a model waxy oil suggests that the situation for waxy crude oils strongly differs from that for thixotropic colloidal suspensions. Indeed, for that model waxy oil a qualitatively similar macroscopic behavior was observed (strong hysteresis in a sweep test as in Fig. 1) but it was shown that after the initial breakage of the structure requiring a high stress the fluid can flow steadily in a wide range of shear rates without exhibiting shear banding. This suggests that for waxy oils the restructuring process is negligible in those experiments, and the irreversible destructuring process plays a major role. It also means that, in general, controlled shear rate tests should not be affected by shear banding. The restructuring capacity of this material is discussed below, in Sec. III E.

Here, we imposed a series of constant shear rate values to oil-gel samples that were submitted to the same cooling history as for the above test, originating the same gel structure. That procedure was repeated for shear rate ranging from 0.01 to $100 \mathrm{~s}^{-1}$, and the shear stress response of the material was measured with time. In this test, the critical 
deformation, where the solid structure collapses, will be achieved in different times according to the magnitude of the imposed shear rate. As a consequence, we look at the stress vs strain evolutions for the different shear rate levels (see Fig. 3). Note that since we are using a controlled stress rheometer, it is not able to impose a stable velocity in the first times of the flow, especially when the apparent viscosity rapidly varies. For the shear rates of 3 and $100 \mathrm{~s}^{-1}$, for example, the rheometer could keep them constant only after deformation values of 1 and 10, respectively. It was possible to observe the importance of this effect from the velocity measurements and we could see that in general the velocity becomes stable and equal to the constant imposed value just after the stress peak, so that this effect should not affect our data analysis beyond the peak.

The first clear trend that may be observed in the data is that the stress vs deformation curves for low deformations (say below 0.05 ) approximately fall along the same curve (see Fig. 3). This means that the rate of shear does not play a significant role here, which indicates that we are in the solid regime. The stress then reaches a maximum value (peak) which depends on the shear rate and finally continuously decreases. The stress peak thus likely marks the transition from the solid to the liquid regime. The fact that the critical deformation associated with this transition (position of the peak) increases with the shear rate is possibly due to a stronger role of viscous effects at higher shear rates, but here we will not attempt to further elaborate on that point.

In the liquid regime, the shear stress undergoes an impressive decrease up to two orders of magnitude, and apparently achieves stable levels for high shear rates. Long transient periods were also observed by Rønningsen (1992) and Zhao et al. (2012). This strong shear stress decrease indicates the rapid and significant material destructuration.

It is worth noting the similarity of the stress vs strain curves in logarithmic scale, which means that they can be superimposed by an appropriate vertical translation. Note that since the apparent viscosity vs deformation curves are obtained by dividing the stress curves by a constant factor, a similar analysis applies with such curves, as shown in the inset of Fig. 3. Those arbitrary factors, used only to show the similarity between the curves, are power-law of the shear rate (numerically, the apparent viscosity was multiplied by $0.002 \dot{\gamma}^{0.75}$ ). This suggests that the viscosity level is given by the applied shear

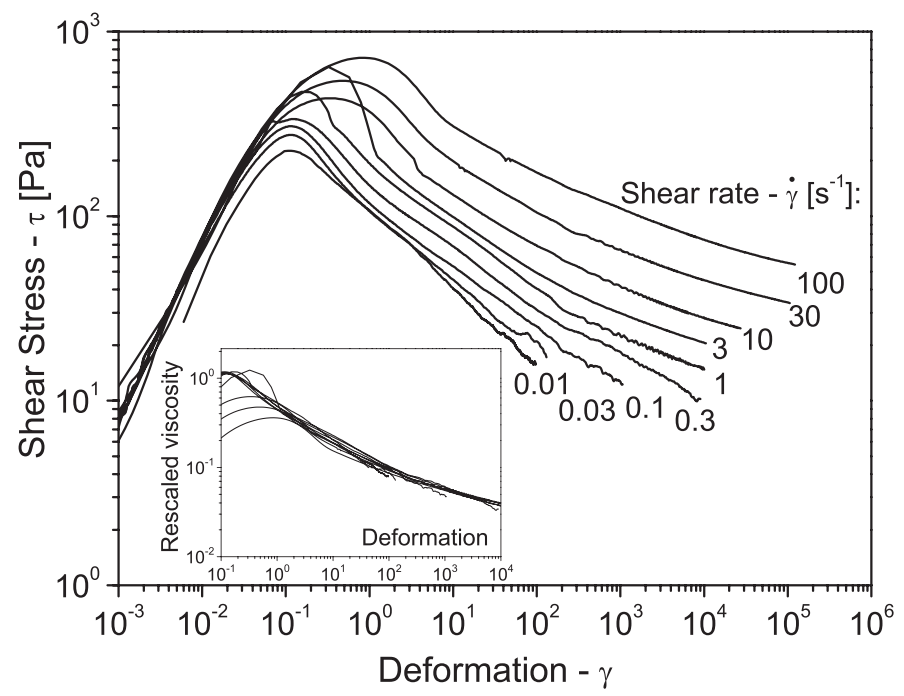

FIG. 3. Shear stress vs deformation response of crude oil A cooled at rest for different imposed shear rate values. The inset shows the apparent viscosity rescaled by arbitrary factors as a function of the deformation. 
rate but the evolution of this viscosity depends only on the total deformation undergone by the fluid from the test beginning. For such a series of tests starting from the same initial state this means that the destructuring rate does not depend on the shear rate but evolves only with the deformation. A higher shear rate will change the material structure state faster only because it deforms the material faster, but the "quantity of deformation" needed to go from a high structure state to the equilibrium is independent of the shear rate level. That point shall be confirmed by the apparent viscosity analysis discussed in detail in Sec. III C.

\section{Sudden shear rate changes}

The above assumption concerning a single structure state associated with a given deformation can be tested by imposing sudden shear rate variations and looking at the variations in apparent viscosity. Considering the similarity of the apparent viscosity curves (see inset of Fig. 3), the fluid should respond to a velocity change by directly moving (by a vertical translation) from the viscosity vs deformation curve associated with the initial shear rate to that associated with the new shear rate value.

In order to check that, we carried out tests by first imposing a given shear rate and then changing it in two steps, one increasing of one decade and a second step returning to the initial shear rate value. During the first stage, the viscosity vs strain curve superimposes the constant shear rate curve obtained previously, thus demonstrating the reproducibility of the data (see Fig. 4). Then for each step of shear rate, we have an excellent agreement with the above assumption as long as the deformation is not too large: The dotted or dashed lines instantaneously drop to the viscosity curve associated with a constant (higher) shear rate from the flow beginning and then follow this curve (see Fig. 4). This tends to confirm that the state of structure reached at a given deformation under a flow at a small shear rate is the same as that reached for the same deformation under higher shear rate flow.

For large deformations, say more than about 100, after a sudden change in shear rate the viscosity does not reach immediately the upper or lower curve. This effect progressively increases for larger deformation, as illustrated in the case of shear rate reductions

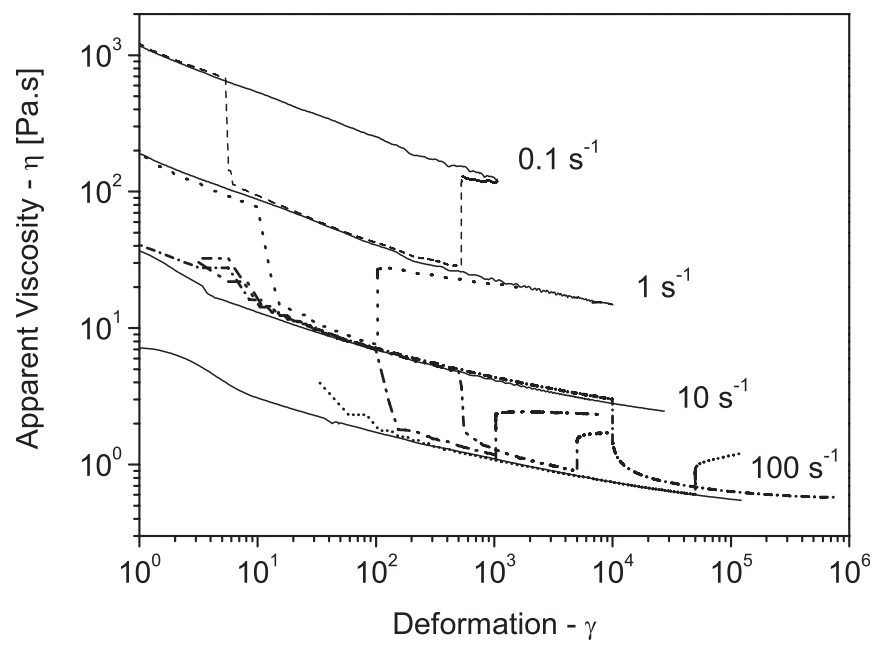

FIG. 4. Apparent viscosity vs deformation for the tests of Fig. 3 at different imposed shear rates (continuous lines). The dashed, dotted, or dashed-dotted lines correspond to independent tests of stepwise changes in shear rate. 
from 100 to $10 \mathrm{~s}^{-1}$. Here, the apparent viscosity tends to remain approximately constant, despite the deformation increase, and it is at a level significantly below the continuous curve associated with the corresponding constant shear rate curve. That means that the structure will a priori never recover that associated with a steady flow at the smaller shear rate, even after a very long time of flow.

In Fig. 4, another interesting behavior can be observed at large deformations. At the shear rate change from 10 to $100 \mathrm{~s}^{-1}$ at $10^{4}$ strain units, for example, the apparent viscosity exhibited an instantaneous reduction, similar to the low deformation step changes but without achieving the corresponding curve of the new shear rate level. That instantaneous jump was followed by a much slower progressive viscosity evolution, apparently toward the curve of constant shear rate of $100 \mathrm{~s}^{-1}$.

Those results mean that the structure state evolution essentially depends on deformation at sufficiently small deformations and significantly depends on the shear rate for large deformations. The latter effect is typical of thixotropy: In a shear rate step, the viscosity takes time before reaching steady state [see Dullaert and Mewis (2006)]. However, here the process seems different: After a large deformation at a high shear rate (e.g., $100 \mathrm{~s}^{-1}$ ) and a jump to $10 \mathrm{~s}^{-1}$ the viscosity rapidly reaches a plateau which is very distant from the curve of constant shear rate at $10 \mathrm{~s}^{-1}$ and would hardly join it even after very long time of flow. Thus the intense shear at $100 \mathrm{~s}^{-1}$ has irreversibly destructured the material, which presents a negligible restructuring capacity with respect to the overall viscosity change during that experiment.

The experiments of step changes in shear rate revealed that there is a same systematic response of the same structure state to different shear rates only at low deformations. However, at high deformations shear rate and deformation cannot be treated as independent variables, as the material does not follow anymore the constant shear rate destructuring curves.

\section{Obtaining the "equilibrium state"}

We have seen above that the fluid progressively gets destructured when submitted to a constant shear rate. Nevertheless, the equilibrium structure, i.e., associated with a steady state flow, is not reached even after $10^{5}$ strain units. Here, we consider that the material has achieved the equilibrium state, for a given shear rate, when the shear stress does not change with time. In order to approach faster the effective equilibrium state of each shear rate level, we will consider the conclusions of Mendes et al. (2014). In that work, it is shown that the equilibrium state for a given temperature history is independent of the flow history when the shear rate is the maximum shear rate experienced by the material. It suggests that a single experiment is sufficient to find the equilibrium states of various shear rates levels if the shear rate is stepwise increased. Thus, a solution for achieving faster the equilibrium state could consist in starting by imposing a flow at low shear rate during the cooling process. We carried out such test by first imposing a low shear rate (namely, $0.1 \mathrm{~s}^{-1}$ ) during the cooling phase from 60 to $4{ }^{\circ} \mathrm{C}$ at $-1{ }^{\circ} \mathrm{C} / \mathrm{min}$ and keeping that shear rate at the constant temperature of $4{ }^{\circ} \mathrm{C}$ for a significant time $(3 \mathrm{~h})$ [see schematic procedure in Fig. 5(a)]. After such procedure, the steady state flow condition seems to be reached [see Fig. 5(b)], i.e., the stress has reached a plateau. If now we increase the shear rate in steps, at each equilibration step that will be the maximum historical shear rate, hence independent of the previous flow. Indeed, when the shear rate is increased after equilibrium in the previous level, the stress first increases and then progressively decreases toward a new equilibrium state. This process repeats itself at each shear rate stepwise increase (see Fig. 5). For these new levels the time of flow, although already 
(a)

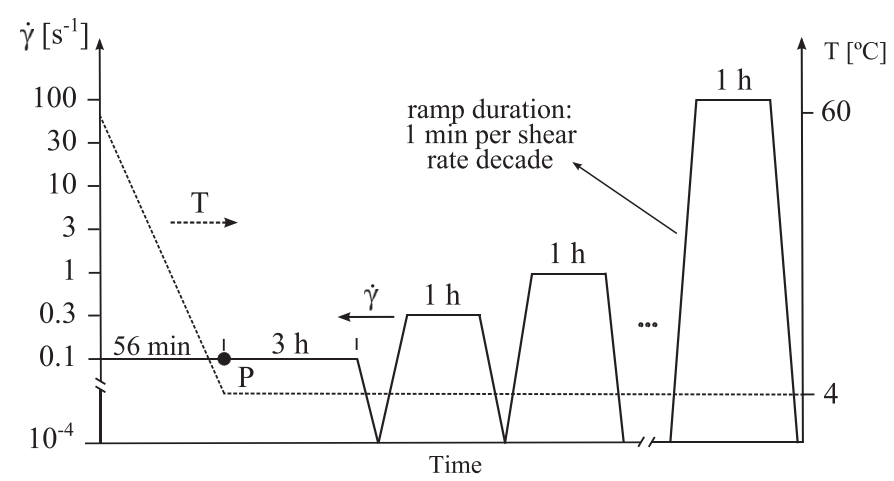

(b)

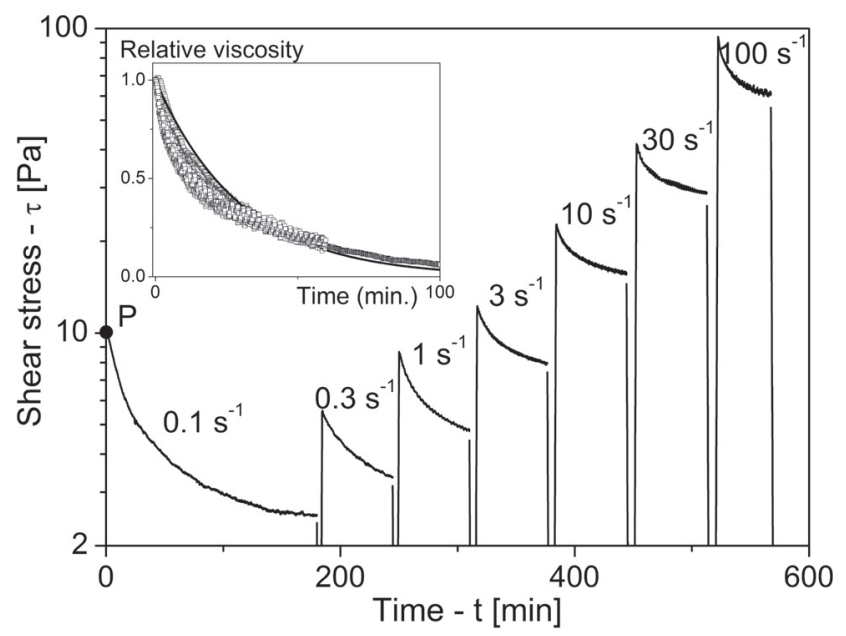

FIG. 5. (a) Schematic procedure used for cooling and imposed shear rate histories. The cooling was under a shear rate of $0.1 \mathrm{~s}^{-1}$. Shear rate is stepwise increased after achieving equilibrium at the previous level, (b) the measured shear stress of crude oil A from the end of cooling period, marked by the point "P." The inset shows the relative viscosity difference to the equilibrium (see definition in text) variations as a function of time for the different shear rate steps (symbols). The continuous dark line in the inset is a model of the type $\left(\eta-\eta_{e q}\right) /\left(\eta_{0}-\eta_{e q}\right)=\exp \left(-t / t_{0}\right)$, where $t_{0}$ is a characteristic time.

large in practice, does not make it possible to reach the exact equilibrium state. It is always difficult to decide whether the equilibrium has been reached, which often leads to arbitrary criteria [e.g., variations smaller than $5 \%$ over three successive periods of $30 \mathrm{~s}$ as used by Dimitriou and McKinley (2014)]. Here, we follow another approach based on the observation that the stress vs time curves obtained after the different steps of shear rate (see Fig. 5) have apparently a similar shape. Thus, we consider the relative viscosity difference to the equilibrium, i.e., $\eta-\eta_{e q} / \eta_{0}-\eta_{e q}$, which $\eta_{0}$ is the apparent viscosity at the initial time (starting at the new shear rate level) and $\eta_{e q}$ is the (unknown) apparent viscosity at equilibrium at this same shear rate level, and we plot this relative viscosity as a function of time. Here, we postulate that $\eta-\eta_{e q} / \eta_{0}-\eta_{e q}$ is a function of time only, and we fit the value for $\eta_{e q}$ so that the curves effectively fall along the same master curve (see inset of Fig. 5).

The values for $\eta_{e q}$ obtained by that procedure are typically on the order of $10 \%$ smaller than the last value obtained at the end of each step.

Let us now look at the (equilibrium) stress $\left(\tau_{e q}=\eta_{e q} \dot{\gamma}\right)$ reached in such a procedure and compare it to the values obtained when starting to impose a flow after a cooling at 
rest. Since we have strong variations (of more than one order of magnitude) of the stress with the deformation (see Fig. 3), it is interesting to look at the stress reached for each shear rate after different deformations. We first can remark that the shear stress vs shear rate curves obtained after different deformations exhibit a similar shape, as shown in Fig. 6. That is a consequence of the apparent similarity of the stress vs deformation curves observed in Fig. 3. Those curves shift downward when the deformation increases, approximately by a constant factor when the deformation is multiplied by 10 . Under these conditions, it seems likely that the stress vs shear rate curve will get very close to the curve representing the equilibrium states after cooling under shear, which is situated at a relatively short distance below the largest iso-deformation curve deduced from our measurements (see Fig. 6). We could fear that the very different flow and temperature history would induce a different structure from that obtained by cooling at rest followed by shear. This does not seem to be the case here. Such a conclusion is also supported by the experiments of Mendes et al. (2014), who showed with the help of MRI velocimetry that for a model waxy oil the same steady state flow curve is obtained after a long time of flow following a cooling under shear or a cooling at rest.

\section{E. Behavior "at equilibrium"}

It was suggested above that the destructuration at high shear rates after a long period of flow (large deformations) could be partly irreversible, the viscosity being unable to get back to its equilibrium level at a lower shear rate (see Fig. 4). In order to clarify this aspect, we first look at the rheological behavior of the fluid when it has reached its equilibrium state. In that aim, between each increasing step at higher shear rate in the above experiment (after cooling under shear) the shear rate was reduced to a very low value before being increased again up to the next step level [see Fig. 5(a)]. Those down and up ramps starting at each equilibrium state, corresponding to a historical maximum shear rate, shall reveal the fluid behavior while the shear rate is below that historical maximum. As the ramps rate of decrease and increase may play a role in the measured behavior due to reversible restructuring effects, two additional experiments were set to evaluate the

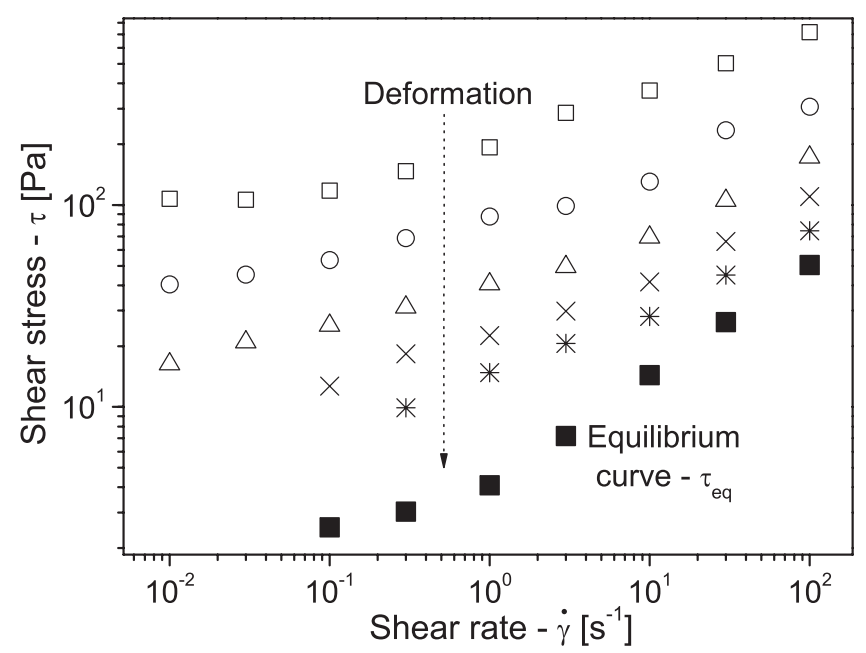

FIG. 6. "Iso-deformation" shear stress vs shear rate curves measured at constant shear rate. Open symbols correspond to data of Fig. 3 at different total deformations: 1 ( $\square$ - squares), 10 ( $\bigcirc$ - circles), 100 ( $\triangle$-triangles), $1000((\times-$ crosses $), 10000(*$-stars). Full squares $(\square)$ are the equilibrium values determined from data of Fig. 5(b) (see text). 
material restructuring capacity during equilibration steps at lower shear rates and during aging at rest.

First, following the procedure in Fig. 5(a), it seems that the shear rate ramps for values below the corresponding shear rate equilibrium point result in an apparent simple liquid behavior (see Fig. 7): The shear stress monotonically decreases with the shear rate reduction and the curves do not exhibit a yield stress, but follow a nearly Newtonian behavior. Moreover, the increasing shear rate curves superimpose to the respective decreasing curves, without changing the fluid behavior within the duration of the ramps ( 1 min per shear rate decade). Those results show that after having reached the equilibrium condition at a given shear rate, the material shows a linear relationship between stress and shear rate over the timescale that the ramp in shear rate occurs, i.e., it behaves as a simple liquid, as it will be discussed next. Furthermore, a new liquid behavior, associated with a smaller viscosity, is reached at each new shear rate level. That means that this behavior of the fluid depends on the level of the maximum shear rate experienced by the fluid; if the fluid is sheared at a higher shear rate than before, its viscosity decreases.

The superimposition of the decreasing-increasing stress vs shear rate curves around some equilibrium suggests that thixotropic effects are weak when it has reached its equilibrium state associated with a given shear rate. In order to confirm that we have carried out tests to look at restructuring effects after strong shear. In that aim, we performed intermediate equilibration steps during a shear rate ramp down test departing from the equilibrium state at $100 \mathrm{~s}^{-1}$, i.e., an unstructured state. At the equilibration steps, the restructuring flow at different shear rate levels was measured. The experimental procedure of this test consists in cooling a crude oil A sample from 60 to $4{ }^{\circ} \mathrm{C}$ at $-1{ }^{\circ} \mathrm{C} / \mathrm{min}$ and shear rate of $100 \mathrm{~s}^{-1}$. This shear rate was kept constant for more $75 \mathrm{~min}$ after the sample reached $4{ }^{\circ} \mathrm{C}$ in order to achieve a stable shear stress value. Next, the shear rate was decreased with a continuous ramp to $10 \mathrm{~s}^{-1}$. At this new shear rate level, we waited for equilibration of the shear stress value. This process continued to the shear rates of

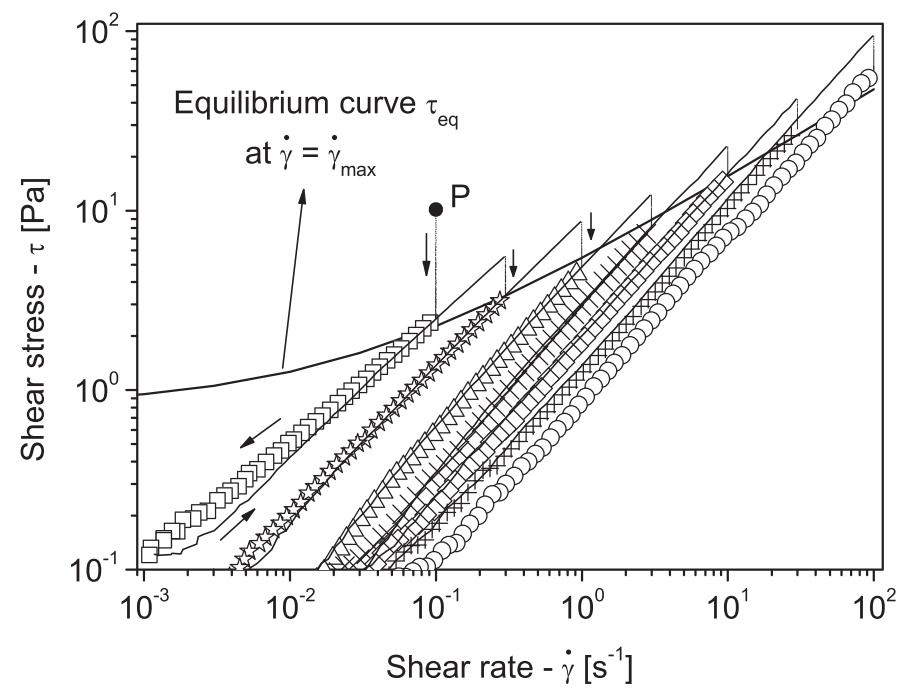

FIG. 7. Shear stress, from the end of the cooling period at an apparent shear rate of $0.1 \mathrm{~s}^{-1}$, marked by the point "P" measured with imposed shear rate according to the procedure of Fig. 5(a). The data are the same as in Fig. 5 (b). Continuous curves correspond to increasing shear rates and symbols to decreasing shear rates. The vertical dashed lines are the stabilization steps. The increasing curves apparently show constant viscosity even after passing by the previous $\dot{\gamma}_{\text {Max }}$. After stress stabilization, at the new $\dot{\gamma}_{\text {Max }}$, the decreasing curves show liquid behavior with lower viscosity for higher $\dot{\gamma}_{\text {Max }}$. 
1 and $0.1 \mathrm{~s}^{-1}$. After this last equilibration step, the shear rate was ramped down to $10^{-4} \mathrm{~s}^{-1}$.

The results presented in Fig. 8 compare the shear stress measured according to the procedure above with the shear rate ramp down (ramp rate of 1 min per decade) from 100 to $10^{-4} \mathrm{~s}^{-1}$ presented in Fig. 7 , represented by circles in both figures. The first aspect to be highlighted in this comparison is that both shear rate ramps starting at $100 \mathrm{~s}^{-1}$ are very similar over the 4 decades of shear stress, despite having completely different flow histories. Important differences appear only for shear stress and shear rate below $0.1 \mathrm{~Pa}$ and $0.1 \mathrm{~s}^{-1}$, the lowest decades. This confirms the concept that the equilibrium state at the historical maximum shear rate experienced by this material is independent of the flow history. It can be also seen in Fig. 8 that the oil restructuring capacity is negligible at the 10 and $1 \mathrm{~s}^{-1}$ steps. The inset in Fig. 8 shows the evolution of the shear stress with time in the equilibration steps at constant shear rate. The most significant structure recover was observed at the shear rate of $0.1 \mathrm{~s}^{-1}$, where the shear stress approximately doubled its value before achieving stable flow at around $6000 \mathrm{~s}$.

Finally, the decreasing shear rate ramp seems a good approximate method for assessing the oil-gel steady state flow curve for shear rates below a given state of equilibrium. Good agreement is obtained during the ramp down until the shear rate of $1 \mathrm{~s}^{-1}$. The most important relative difference was measured at $0.1 \mathrm{~s}^{-1}$, which was of the order of $0.1 \mathrm{~Pa}$. This means that restructuring during flow (after a decreasing step of shear rate) is generally negligible, mainly when compared to the structure state presented by the material just after static cooling.

In order to evaluate the material restructuring capacity at rest, additional shear ramps cycles were performed. In these tests, a first ramp up and down cycle, departing from a statically cooled oil-gel, was executed followed by different holding times at rest before running the second ramps cycle. Figure 9(a) shows the schematic procedure of those tests, where during the cooling phase and holding times, the material is at rest.

Figure 9(b) presents the measured shear stress curves for the increasing shear rate ramps for different holding times between cycles. It appears that for the first cycle (filled

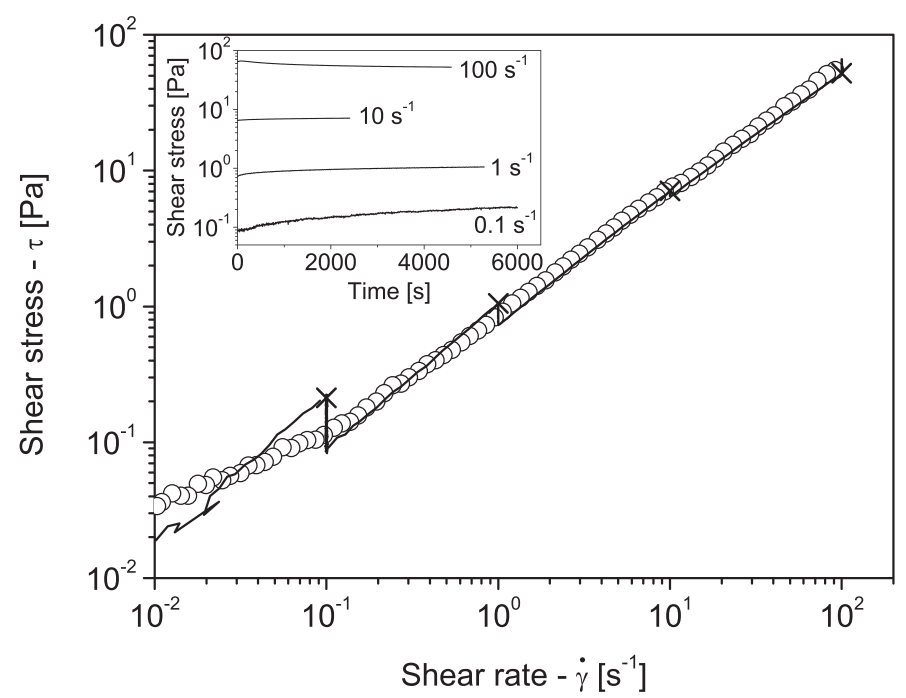

FIG. 8. Comparison between the shear rate ramp down starting at $100 \mathrm{~s}^{-1}$ of Fig. 7 (circles) and a ramp down test also starting from the equilibrium condition at $100 \mathrm{~s}^{-1}$ but with intermediate equilibration steps at 10,1 , and $0.1 \mathrm{~s}^{-1}$. The shear stress variation during the equilibration steps is shown in the inset. 
(a)

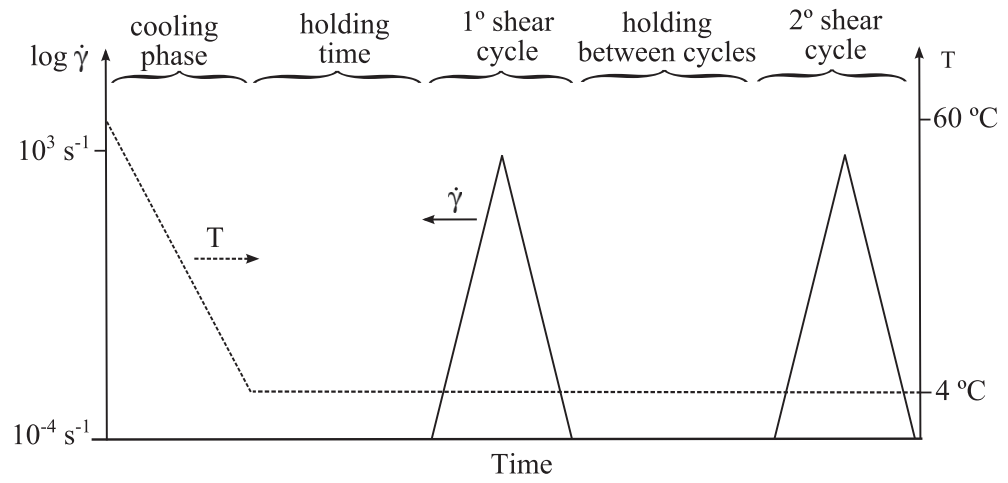

(b)

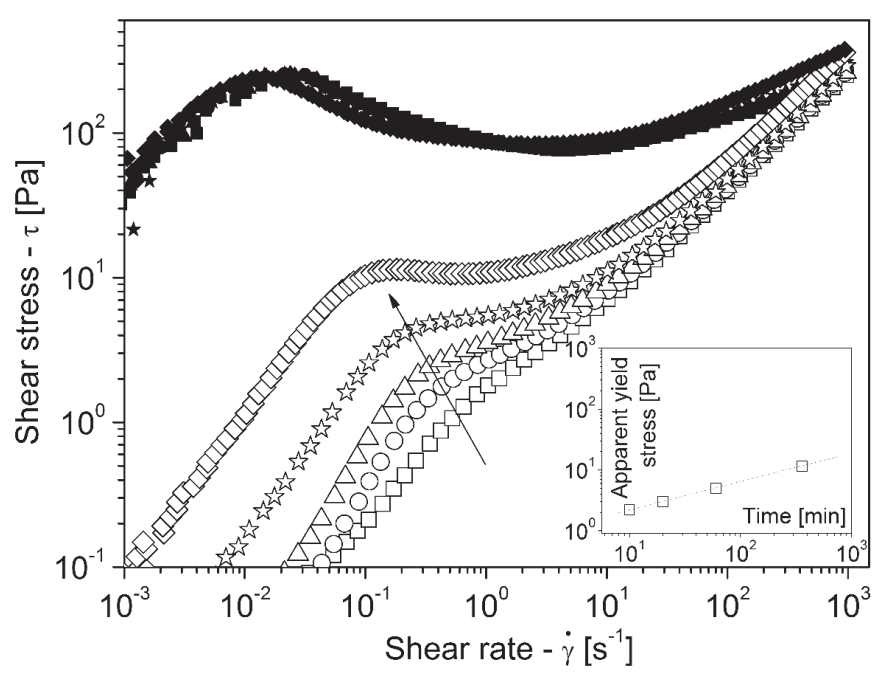

FIG. 9. (a) Schematic temperature and imposed shear rate for analyzing the fluid recovery capacity after shear. The holding time between cycles was changed in each test, from 0 to $360 \mathrm{~min}$. (b) Measured shear stress of crude oil A for holding times of 0 ( $\square$-squares), 10 ( $\bigcirc$ - circles), 20 ( $\triangle$ —triangles), $60 \mathrm{~min}$ ( $\lesssim$ —stars), and $360 \mathrm{~min}(\diamond$-diamonds). Only the up ramps are shown, filled symbols correspond to the first ramp, open symbols to the second one.

symbols), executed after the initial cooling, the curves superimpose themselves, indicating a good repeatability of this experiment. Then the ramp up in the second shear sweep cycle (open symbols) without holding time between cycles is very similar to the previous ramp down, observed in Fig. 1. The curves for increasing holding times present the same initial slope, but the shear stress increase happens progressively at lower shear rates. Increasing intermediate regions with lower slopes are observed. As the shear rate increases, the curves finally superimpose.

It is possible to see in Fig. 9(b) that crude oil A exhibits a very slow restructuring process. In order to quantify it, we evaluated the apparent yield stress of the ramp up of the second shear cycle in function of the holding time at rest between cycles [see inset of Fig. 9(b)]. The apparent yield stresses were inferred through the change in slope of the increasing curves. Specifically, two regions are fitted using a straight line and their intersection point is taken as the yield stress. The apparent yield stress increases as a small power law of the time at rest and it is still 30 times smaller than the initial yield stress after $6 \mathrm{~h}$ at rest. Moreover, an extrapolation the line fitted to the data in the inset of Fig. 9(b) indicates that the recovery of the initial yield stress would need about $9000 \mathrm{~h}$ at 
rest, about one year. Typically, within our framework, realistic time scales are not larger than several tens of hours. Hence, restructuring will remain small in terms of apparent yield stress, which will never recover its initial value. In practical terms, for a pump system that is able to start the flow overcoming the apparent yield stress of the first ramp up, restructuring in terms of apparent yield stress would not be a problem at all.

\section{MODELING}

\section{A. Model}

The rheological behavior of waxy crude oils as it appears from the various tests presented in this work seems rather complex, and does not fall in a class of materials exhibiting a well-known type of constitutive equation. In particular, it clearly does not correspond at all to the usual thixotropic behavior since one major trend is that when starting from a cooling at rest we have a strong, almost irreversible, destructuration of the material. Under these conditions, we will keep only the fundamental rheological characteristics of these materials as we have identified them (see above) and which are expressed as follows:

- Once the oil-gel structure is broken from rest, by achieving a critical shear stress or a critical deformation, a strong destructuring flow takes place, irreversibly changing the fluid structure state;

- In a first stage, this destructuring essentially depends on the deformation undergone by the fluid from its solid-liquid transition, and in a second stage it also depends on the flow characteristics (shear rate);

- After being sufficiently sheared, an equilibrium state is achieved for a given shear rate when it is the maximum shear rate experienced by the fluid;

- From that equilibrium state, the behavior can be essentially considered as that of a simple liquid if the fluid is not sheared beyond that maximum "historical" shear rate;

In that context, the behavior in the solid regime will not be considered in detail. We will simply assume that when cooled at rest, the material is a viscoelastic solid, i.e., the stress is proportional to the deformation and the shear rate: $\tau=G \gamma+\mu_{S} \dot{\gamma}^{n}$, and turns to a liquid for a critical deformation $\gamma_{c}$, so that it presents a yield stress $\tau_{c}=G \gamma_{c}$ when deformation rate tends to zero. In this approach, the critical parameter is the yield stress, which is the stress to be overcome for starting flowing. The critical deformation, which here appears to be around 0.15 (see Fig. 3), is also a relevant parameter of the fluid, which can be determined from the position of the stress peak at low shear rates. We will not further look at the detailed behavior in the solid regime assuming it does not play a major role in the start-up flow once the solid-liquid transition is defined and solid deformations are much smaller than 1. However, it is possible to study or model in more detail the behavior in the solid regime [see, for example, de Souza Mendes et al. (2011) and Dimitriou and McKinley (2014)].

In the liquid regime, the behavior is described by the apparent viscosity, $\eta$. At equilibrium state, the fluid may be considered as a first approximation as Newtonian, i.e., $\eta$ is constant, in a range of shear rates that depends on the flow history. Moreover, the steady state value of $\eta$ depends on the flow history. A complete mapping of this viscosity value as a function of flow history would be difficult. Nevertheless, we may refer to the equilibrium behavior obtained after a very long flow under a given shear rate which is also the maximum shear rate undergone by the fluid, since it has started to be cooled. The viscosity corresponding to that equilibrium behavior may be represented by a HB model (see Fig. 7)

$$
\eta_{e q}=\left(\tau_{0, e q}+k_{0, e q} \dot{\gamma}_{\max }^{n}\right) / \dot{\gamma}_{\max }
$$


where $\tau_{0, e q}, k_{0, e q}$, and $n$ are constants for the given temperature. In that equilibrium state, the fluid exhibits a constant viscosity defined by Eq. (1) as long as we now impose from this state a shear rate smaller than $\dot{\gamma}_{\max }$. So, for $\dot{\gamma}<\dot{\gamma}_{\max }$ we have $\tau=\eta_{e q}\left(\dot{\gamma}_{\max }\right) \dot{\gamma}$ As a consequence, despite the same format, it cannot be considered that the crude oil follows a HB model, since in Eq. (1) each stress vs shear rate pair is associated with a specific irreversible state of destructuring, whereas for a usual $\mathrm{HB}$ behavior it is possible to move along this curve by increasing or decreasing the shear rate. It should also be said that, by assuming a constant viscosity for $\dot{\gamma}<\dot{\gamma}_{\text {max }}$, the this model does not account for the small restructuring effects observed for shear rates below $1 \mathrm{~s}^{-1}$ in Fig. 8.

In the mathematical description presented here, the shear rate variable $\dot{\gamma}$ is considered to be the modulus of the strain rate tensor. Moreover, this modeling accounts only for increasing deformation, i.e., the deformation $\gamma$ is calculated by the integration of the shear rate modulus in time.

In view of describing the transient characteristics of this fluid, some aspects of the destructuring flow can be highlighted. We have seen that during a first phase of flow there is a similarity of the shear stress vs deformation curves (see Fig. 3), which was confirmed by the similarity of the shear stress vs shear rate curves at fixed deformation (see Fig. 6). In addition, we have considered that the stress tends toward an equilibrium value for sufficiently large deformations. As a consequence, we can look at the evolution of $\tau-\tau_{e q}$ as a function of the deformation. It appears that for the different shear rates the different $\tau-\tau_{e q}$ vs $\gamma$ curves are parallel in a logarithmic scale. This means that $\tau-\tau_{e q}$ is proportional to $\gamma^{-m}$ by a factor which depends on the shear rate. As the shear stress vs shear rate curves for iso-deformations in Fig. 6 can be represented by HB-type equation, that factor may be expressed in the form $\tau_{0}+k \dot{\gamma}^{n}$. The parameters $\tau_{0}$ and $k$ are different from $\tau_{0, e q}$ and $k_{0, e q}$. The first two parameters express the shear stress difference to its equilibrium condition, defining the proportionality between $\tau-\tau_{e q}$ and $\gamma^{-m}$, while $\tau_{0, e q}$ and $k_{0, e q}$ represent the equilibrium condition itself. In Fig. 10, we show how the $\tau-\tau_{e q}$ vs $\gamma$ curves effectively fall along a single curve when rescaled by this factor. Finally, for a deformation between 1 and $10^{4}$ the apparent viscosity may be represented by the following expression, valid within 20\% when a constant shear rate is applied (continuous lines in Fig. 4):

$$
\eta=\eta_{e q}+\left(\tau_{0}+k \dot{\gamma}^{n}\right) \gamma^{-m} / \dot{\gamma}
$$

It is worth emphasizing that, as it has been established, this equation only describes the rheological behavior of the fluid when a constant shear rate is applied just after the cooling period when the material has reached a gel behavior, which is obviously not the general case. The simplest approach for generalizing the model would assume that this equation gives the instantaneous apparent viscosity under a given shear rate after any flow history leading to a total deformation $\gamma$. This approach is the equivalent of considering that the material reaches a given state of structure which depends only on the total deformation, and this state of structure has an impact on the shear stress through the factor $\gamma^{-m}$ in Eq. (2). This description appears to be in agreement with data for strong shear rate variations (up or down) as long as the deformation is sufficiently small (see Fig. 4).

However, as the deformation increases and if the shear rate changes with time, this description does not correspond any more to the observations (see Sec. IIIC). In that case, the fluid only tends progressively to the viscosity given by Eq. (2) (see Fig. 4), as if it was an attractor point. For very large deformation and shear rate decrease, it may even never reach this curve, remaining at a smaller viscosity [see Figs. 4 and 5(b)]. Note that in that case, for a shear rate jump [see the inset of Fig. 5(b)], initially the apparent 


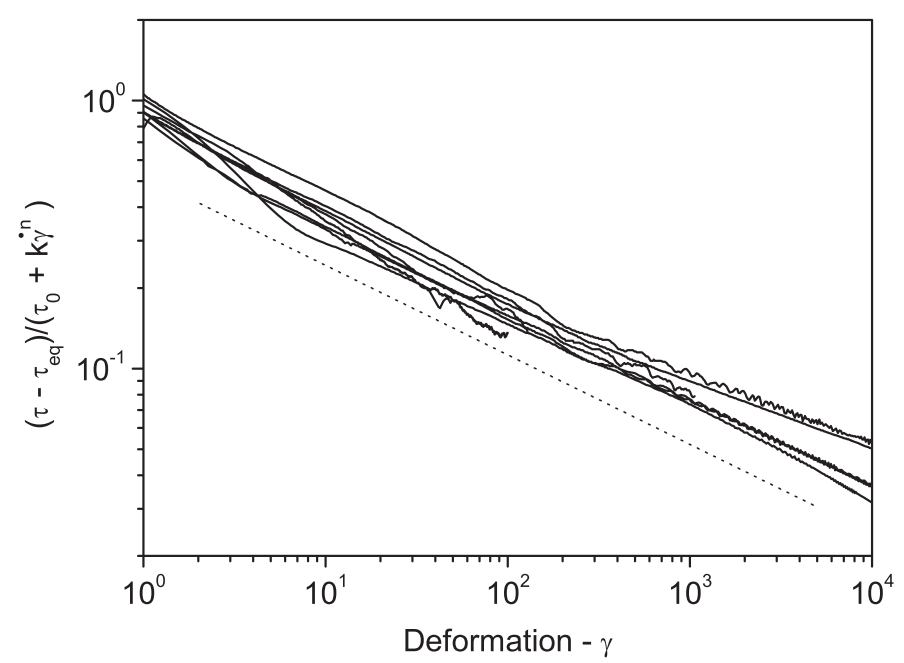

FIG. 10. Variation of the relative stress distance from the equilibrium state, scaled by the factor $\tau_{0}+k \dot{\gamma}^{n}$ as a function of deformation for start up flows just after static cooling at different shear rates (data of Fig. 3).

viscosity does not change and then evolves toward the equilibrium value with a timing for reaching the equilibrium level which may be represented by an equation of the form: $\left(\eta-\eta_{e q}\right) /\left(\eta_{0}-\eta_{e q}\right)=\exp \left(-t / t_{0}\right)$, where $t_{0}$ is a characteristic time. That equation is equivalent to the form $d \eta / d t=-\left(\eta-\eta_{e q}\right) / t_{0}$.

To sum up: At low deformations for complex flow history the fluid evolves almost instantaneously through Eq. (2); as the deformation increases, if the shear rate is changed viscosity takes some time to reach the value predicted by Eq. (2) for the current shear rate level. But when that level has been reached, the viscosity once again evolves with deformation according to the prediction of Eq. (2) as long as the shear rate is kept constant; at large deformations, if the shear rate is increased, the time for reaching the equilibrium is very long but follows a simple kinetics well represented by equation $d \eta / d t$ $=-\left(\eta-\eta_{e q}\right) / t_{0}$ defined above; at large deformations, close to the equilibrium state, if the shear rate is decreased the fluid now tends to partly keep its apparent viscosity.

Finally, the behavior of the fluid depends on the flow history in a complex way while our observations necessarily concern simple flow histories. This has several consequences. First, it appears convenient to use the viscosity expression given by Eq. (2) as a reference behavior to describe the fluid viscosity evolution. That expression in particular means that the equilibrium state behavior expected after a long flow under a given shear rate (i.e., more precisely, a very large deformation) corresponds to a Newtonian behavior with a viscosity defined by Eq. (1) for a shear rate below $\dot{\gamma}_{\max }$. Then, in order to describe the time variations of the viscosity as a function of the flow history it is convenient to describe this flow history through two variables here considered independent, namely the deformation and the shear rate. This means that we may write the viscosity kinetic equation as

$$
\frac{\mathrm{d} \eta}{\mathrm{d} t}=\frac{\mathrm{d} \eta(\gamma, \dot{\gamma})}{\mathrm{d} t}=\dot{\gamma}\left(\frac{\partial \eta}{\partial \gamma}\right)_{\dot{\gamma}}+\frac{d \dot{\gamma}}{d t}\left(\frac{\partial \eta}{\partial \dot{\gamma}}\right)_{\gamma}
$$

At this stage, the simplest approach for the first term assumes a behavior of that observed at the approach of the equilibrium curve under constant shear rate, but now with a target viscosity defined by Eq. (2) and that we will write $\eta_{\text {ref }}(\gamma, \dot{\gamma})$, and a 
characteristic time $t_{0}$ varying with the distance from the equilibrium. For the second term, we do not have much information from the experimental data. We will roughly assume that in this expression the variation of $\eta$ with $\dot{\gamma}$ is equal to the variation of $\eta_{\text {ref }}$ with $\dot{\gamma}$ times a factor $\alpha$ depending on the distance to the equilibrium, i.e., $\alpha=1$ at the solid-liquid transition and $\alpha=0$ at equilibrium of the corresponding shear rate. We finally obtain the following kinetic equation:

$$
\frac{\mathrm{d} \eta}{\mathrm{d} t}=-\frac{1}{t_{0}(\eta)} \operatorname{Sup}\left\{0 ;\left(\eta-\eta_{r e f}\right)\right\}_{\dot{\gamma}}+\alpha(\eta) \frac{d \dot{\gamma}}{d t}\left(\frac{\partial \eta_{r e f}}{\partial \dot{\gamma}}\right)_{\gamma}
$$

Note that the $\operatorname{Sup}\{\}$ function in the first term in the RHS ensures that the viscosity will remain constant if the new imposed shear rate is below the state of reference which has been reached at that time. Finally, convenient expressions for $\alpha$ and $t_{0}$ are as follows:

$$
\alpha=\left[\frac{\ln \left(\eta / \eta_{e q}\right)}{\ln \left(\eta\left(\gamma_{c}\right) / \eta_{e q}\right)}\right]^{a} ; \quad t_{0}=1+K \alpha^{-M},
$$

where $\alpha$ is ranged between 0 and 1 and $t_{0}$ is bounded by the characteristic time associated to highly sheared states $\left(t_{0, \max }\right)$ as observed in Fig. 5. $K, M$, and $a$ are constant parameters which may be fitted to our data in the asymptotic cases (very close or very far from the equilibrium) and for transient flows associated with jumps of shear rate.

It is worth noting that while the first term in the RHS of Eq. (4) predicts the viscosity behavior at constant shear rate (such as the continuous lines in Fig. 4 or the viscosity variations in Fig. 5) and bounds its growth in reducing shear rate cases (Fig. 4), the second term allows predicting viscosity instantaneous changes as a result of shear rate instantaneous changes, for both increasing and decreasing shear rate cases (as the viscosity instantaneous variations observed in Fig. 4).

In summary, the model proposed here may be written as in the following equations:

$$
\begin{gathered}
\tau=G \gamma+\mu_{S} \dot{\gamma}^{n} \quad \text { if } \gamma<\gamma_{c}, \\
\tau=\eta \dot{\gamma} \quad \text { if } \gamma \geq \gamma_{c}, \\
\frac{\mathrm{d} \eta}{\mathrm{d} t}=-\frac{1}{t_{0}} \operatorname{Sup}\left\{0 ;\left(\eta-\eta_{r e f}\right)\right\}_{\dot{\gamma}}+\alpha \frac{d \dot{\gamma}}{d t}\left(\frac{\partial \eta_{r e f}}{\partial \dot{\gamma}}\right)_{\gamma}, \\
\eta_{r e f}=\left(\tau_{0, e q}+k_{0, e q} \dot{\gamma}^{n}\right) / \dot{\gamma}+\left(\tau_{0}+k \dot{\gamma}^{n}\right) \gamma^{-m} / \dot{\gamma}, \\
\alpha=\left[\frac{\ln \left(\eta / \eta_{e q}\right)}{\ln \left(\eta\left(\gamma_{c}\right) / \eta_{e q}\right)}\right]^{a}, \quad 0 \leq \alpha \leq 1, \\
t_{0}=1+K \alpha^{-M}, \quad t_{0} \leq t_{0, \max },
\end{gathered}
$$

where $\tau_{0, e q}, k_{0, e q}, \tau_{0}, k, n, m, \gamma_{c}, a, K, M$, and $t_{0, \max }$ are constant positive parameters of the model.

The model can be used for simulating imposed shear rate cases, for example, as follows: While in the solid regime, the shear stress is calculated by the viscoelastic solid model given by Eq. (5). The deformation $\gamma$ is known from the integration of the shear rate with time. When the critical deformation $\gamma_{c}$ is achieved the material becomes liquid 
and its first apparent viscosity value is assumed to be equal to $\eta_{\text {ref }}$, given by Eq. (8). Thus, now in the liquid regime, the shear stress is calculated by Eq. (6) and the viscosity $\eta$ evolves in time according to Eq. (7).

It is interesting to note that the critical stress for the solid-liquid transition appears in the model by substituting Eq. (8) in Eq. (6), taking $\gamma=\gamma_{c}$ and the limit $\dot{\gamma} \rightarrow 0: \tau_{c}$ $=\tau_{0, e q}+\tau_{0} \gamma_{c}^{-m}$. In order to have the stress continuity in the solid-liquid transition, the solid viscoelastic parameters may be calculated as $G=\tau_{c} / \gamma_{c}$ and $\mu_{S}=k_{0, e q}+k \gamma_{c}^{-m}$.

\section{B. Comparison to experimental data}

Since the structure of the model has been basically deduced from experimental observations and most of the parameters have been fitted to data associated with simple flow histories, a comparison of the model predictions with the data used to fit the model parameters is let to the Appendix. Under these conditions, it is more interesting to look at the predictions of the model in the case of more complex flow histories such as creep tests or progressive variations of the shear rate. Table I presents the parameters of the model for crude oils A and B.

The parameters $\tau_{0, e q}, k_{0, e q}$, and $n$ were determined from the equilibrium curve for $\dot{\gamma}$ $=\dot{\gamma}_{\max }$ in Fig. 7 (also shown in Fig. 6 as black squares). The destructuring flow data at constant shear rate of Fig. 3 were used to find the parameters $\tau_{0}$ and $k$. With the appropriate values, all curves shall have the same relative stress distance to equilibrium, as shown in Fig. 10. This figure provides the value of the deformation exponent $m$. The parameter $a$ was fitted to provide the best average magnitude of the viscosity instantaneous variations due to instantaneous shear rate changes at the discontinuous lines in Fig. 4. The parameters $M$ and $K$ were chosen to fit the progressive viscosity evolution that follows that shear rate change. The characteristic time of that evolution is bounded by a maximum value $t_{0, \max }$ found to be the same for all constant shear rate curves of Fig. 5(b) (destructuring flow close to equilibrium). The critical deformation $\gamma_{c}$ was defined from the maximum shear stresses observed in Fig. 4 (confirmed by creep tests shown in Fig. 2).

Figure 11 shows the comparison between the model predictions and the creep tests presented in Sec. III A. The material response below the critical deformation is captured by the model by calculating the final deformation when the imposed shear stress is below the critical value. For crude oil A, at the cooling conditions described for those tests, the critical stress is $224 \mathrm{~Pa}$. Hence, for imposed shear stresses below that value the model predicts no flow, just finite deformation. For imposed shear stresses above the critical stress value,

TABLE I. Parameters of the model measured for crude oils A and B.

\begin{tabular}{lcc}
\hline \hline Parameter & Crude oil A & Crude oil B \\
\hline$\tau_{0, e q}(\mathrm{~Pa})$ & 0.66 & 8.5 \\
$k_{0, e q}\left(\mathrm{~Pa} \mathrm{~s}^{\mathrm{n}}\right)$ & 4.3 & 39 \\
$n$ & 0.5 & 0.5 \\
$\tau_{0}(\mathrm{~Pa})$ & 103 & 330 \\
$k\left(\mathrm{~Pa} \mathrm{~s}{ }^{\mathrm{n}}\right)$ & 76.3 & 400 \\
$m$ & 0.4 & 0.5 \\
$M$ & 12 & 4.3 \\
$a$ & 0.3 & 0.7 \\
$K(\mathrm{~s})$ & 0.1 & 0.05 \\
$t_{0, \max }(\mathrm{s})$ & 1800 & 2000 \\
$\gamma_{c}$ & 0.15 & 0.1 \\
\hline \hline
\end{tabular}




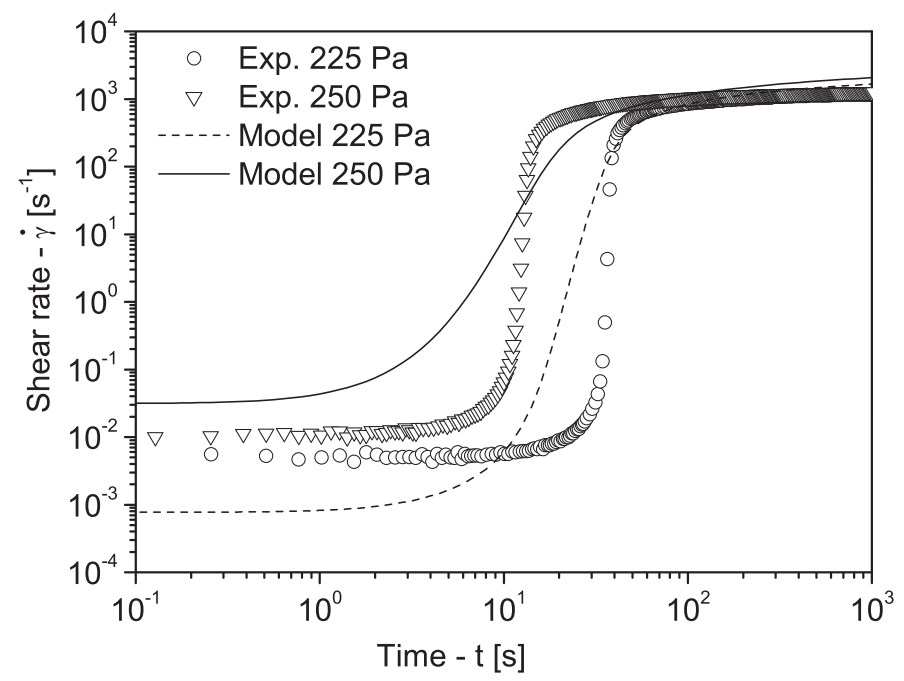

FIG. 11. Model predictions comparison to imposed shear stress experiments with crude oil A. Time is equal to zero at the critical deformation, which is considered to represent the solid-liquid transition and is the minimum of curves in Fig. 2.

the material starts flowing. It begins with a very low shear rate followed by a rapid increase capturing the impressive structure collapse. Then, the shear rate will tend toward its equilibrium value. It is remarkable that the strong structure collapse will allow high shear rate equilibrium values for constant stress cases. As discussed above, the minimum stress required to start the flow is given by $\tau_{c}=\tau_{0, e q}+\tau_{0} \gamma_{c}^{-m}$. If that shear stress is left constant in time, at the equilibrium we will have $\tau_{c}=\eta_{e q} \dot{\gamma}$, i.e., an equilibrium shear rate given by $\dot{\gamma}=\left[\tau_{0} \gamma_{c}^{-m} / k_{0, e q}\right]^{1 / n}$. According to the values of the fitted parameters, in Table I, that equilibrium shear rate obtained when the critical stress is kept constant is $2617 \mathrm{~s}^{-1}$.

The final shear rates are predicted to be higher than the measured values, probably because they are far out of the range of shear rates in the data used to fit the model parameters at steady state flow, resulting in an underestimation of the power coefficient of the shear rate. Also in quantitative terms, the model seems to predict a slow onset of flow for the $250 \mathrm{~Pa}$ curve. In Fig. 11, time equal to zero corresponds to the critical deformation, thus only the material behavior after the solid-liquid transition is compared, since the model does not account for the dynamics in the solid regime. Finally, from a qualitative point of view, we have a good overall agreement of the model predictions with the data.

We now turn to tests in which the imposed shear rate is varied in time. The first test of that type is the sweep test used in Fig. 1, in which the shear rate is increased from 0 to a large value then decreased. We observe (see Fig. 12) that the model (fitted to the data under simpler flow histories) is in good agreement with the experimental data obtained for this typical test, both in the solid and the liquid regime. It is, in particular, interesting to note that it represents the decreasing then increasing parts of the stress vs shear rate curve during the increasing ramp, since this suggests that it properly captures the timing for destructuring under complex flow histories.

In two other cases, starting from rest after static cooling, we imposed a given shear rate during $10 \mathrm{~s}$ then decreased the shear rate from this value to almost 0 in $2 \mathrm{~min}$. We can see that once again the model well represents the stress vs shear rate curves during these transient flows, which confirms our above conclusions about its ability to properly reproduce the characteristic time of destructuring and the viscosity increase with shear rate reduction during the destructuring flow. 


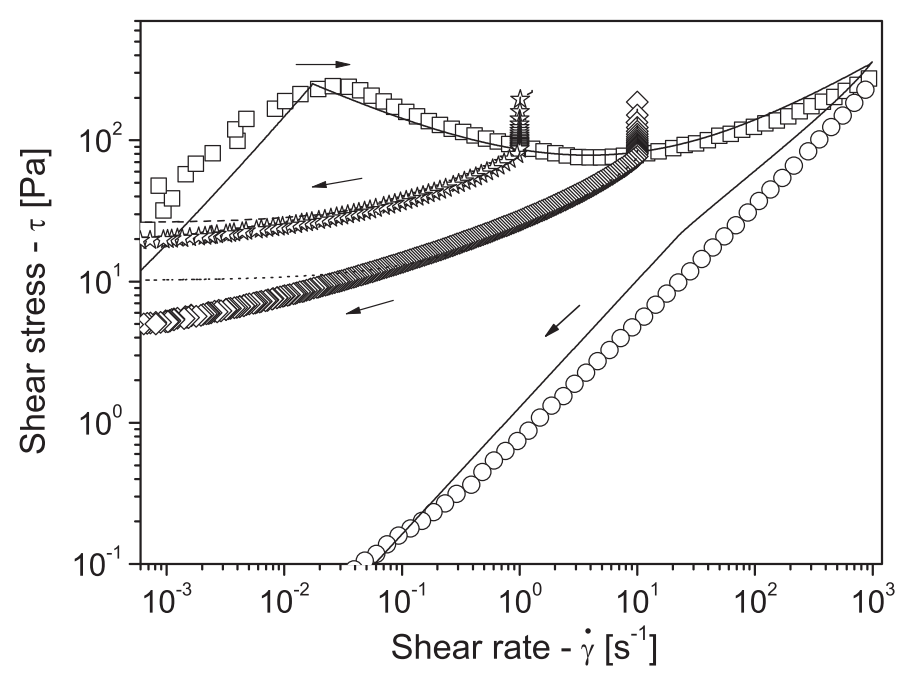

FIG. 12. Experiments with imposed shear rate variations in time and comparison with the predictions of our model (continuous, dashed, and dotted lines) for crude oil A: Shear ramps test (data of Fig. 1) ( $\square$ —squares and $\bigcirc$ - circles), constant shear rate during $10 \mathrm{~s}\left[1 \mathrm{~s}^{-1}\right.$ (放—stars) or $10 \mathrm{~s}^{-1}$ ( $\diamond$-diamonds) ] then logarithmic decrease down to $10^{-4} \mathrm{~s}^{-1}$ in $2 \mathrm{~min}$.

The same conclusions apply when the above experiments are performed with crude oil B. Figure 13 presents the same shear rate ramp test as that of Fig. 12, but with crude oil B. The inset shows the comparison of the creep test with crude oil B imposing a shear stress of $1000 \mathrm{~Pa}$.

We also fitted the Houska model to measured data of crude oil A, according to the fitting procedure suggested by Cawkwell and Charles (1989) and further developed by Hénaut and Brucy (2001). The Houska model is given by

$$
\tau=\tau_{y 0}+\lambda \tau_{y 1}+(k+\lambda \Delta k) \dot{\gamma}^{n}
$$

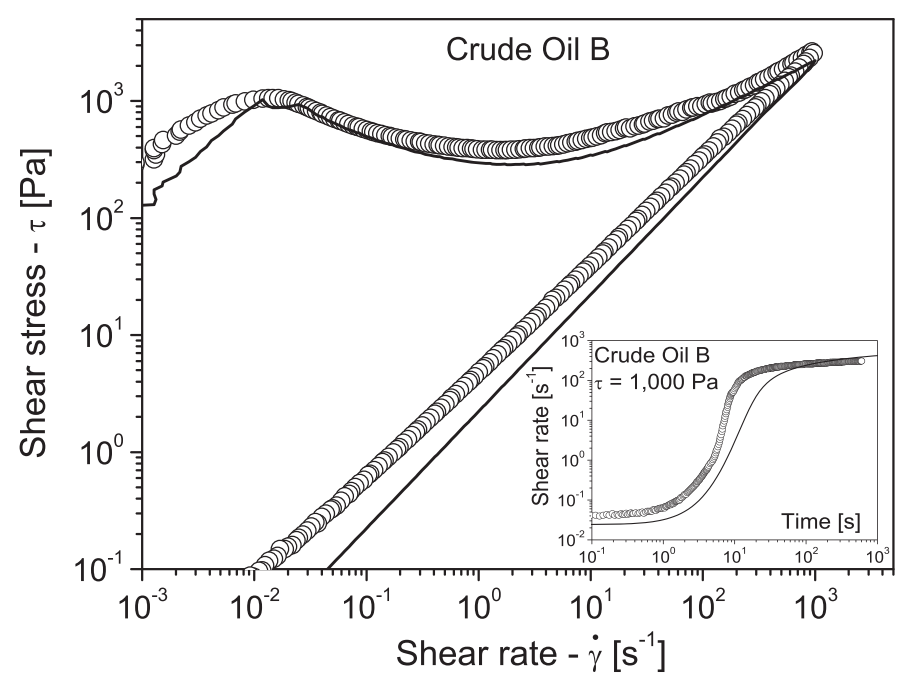

FIG. 13. Comparisons between measured (symbols) and calculated data (continuous line) for crude oil B in the same experiments of Fig. 11 (creep test, in this inset) and Fig. 12 (shear ramp test). 


$$
\frac{d \lambda}{d t}=a(1-\lambda)-b \lambda \dot{\gamma}^{m}
$$

where $\tau_{y 0}$ and $\tau_{y 1}$ are the permanent and time-dependent yield stresses, $k$ and $\Delta k$ are the steady state and time-dependent consistency parameters, $n$ is the power-law coefficient, $a$ is the structure buildup coefficient, $b$ is the break down coefficient, and $m$ is the adjusting parameter for shear rate dependence. $\lambda$ is the structure parameter that is equal to zero when the oil-gel is in a completely destructured state and equal to 1 when the gel is at its strongest state.

The fitting procedure consists of estimating $\tau_{y 0}, k$, and $n$ by measuring the oil flow curve when the crude oil is completely destructured, i.e., after cooling at $-1{ }^{\circ} \mathrm{C} / \mathrm{min}$ and $10 \mathrm{~min}$ of flow at $1000 \mathrm{~s}^{-1}$ at the desired test temperature $\left(4^{\circ} \mathrm{C}\right.$ in our case). The parameters $\tau_{y 1}$ and $\Delta k$ are fitted from the maximum shear stresses measured from imposed shear rates tests from static cooled oil samples. Here, the maximum values of the curves in Fig. 3 were used. The parameters $a, b$, and $m$ are fitted from partial destructured states, i.e., after $1 \mathrm{~min}$ of flow at different shear rate values. The use of more data points from the curves of Fig. 3 did not improve the predictions of the model. Table II presents the values of the parameters found for crude oil A.

Figure 14 presents the comparison of the data obtained with the Houska model with the same measured data of Figs. 11 and 12. Clearly, this model is unable to predict the behavior of the material under such complex flow histories. The discrepancy on the stress values between the model predictions and the data is often larger than one order of magnitude.

\section{TEMPERATURE EFFECTS}

We carried out similar tests with waxy crude oil A now cooled to different temperatures between 6 and $20^{\circ} \mathrm{C}$, which made it possible to determine the relevant rheological parameters identified above. The apparent yield stress and the critical deformation could be measured from creep tests at low shear rates. The yield stress continuously decreases as the temperature increases and tends to zero around $16^{\circ} \mathrm{C}$ (see Fig. 15), which means that beyond $18^{\circ} \mathrm{C}$ the material is a liquid without yield stress. In most of the range where a yield stress exists the critical deformation remains constant around 0.15 .

The equilibrium curves were obtained using the same procedure as above for $4{ }^{\circ} \mathrm{C}$, i.e., the crude oil was cooled under shear at low shear rate and once at the desired temperature, the shear rate was stepwise increased, waiting for the equilibrium condition at each shear rate step. As expected, higher temperatures result in lower shear stresses for the same shear rate (see Fig. 16). The equilibrium curves obtained for the different

TABLE II. Houska model parameters for crude oil A.

\begin{tabular}{lc}
\hline \hline$\tau_{y 0}(\mathrm{~Pa})$ & 0 \\
$\tau_{y 1}(\mathrm{~Pa})$ & 224 \\
$k\left(\mathrm{~Pa} \mathrm{~s}{ }^{\mathrm{n}}\right)$ & 0.237 \\
$\Delta k\left(\mathrm{~Pa} \mathrm{~s}^{\mathrm{n}}\right)$ & 180 \\
$n$ & 0.96 \\
$a\left(\mathrm{~s}^{-1}\right)$ & $2.33 \times 10^{-4}$ \\
$b\left(\mathrm{~s}^{1 / \mathrm{m}}\right)$ & 0.03 \\
$m$ & 0.124 \\
\hline \hline
\end{tabular}



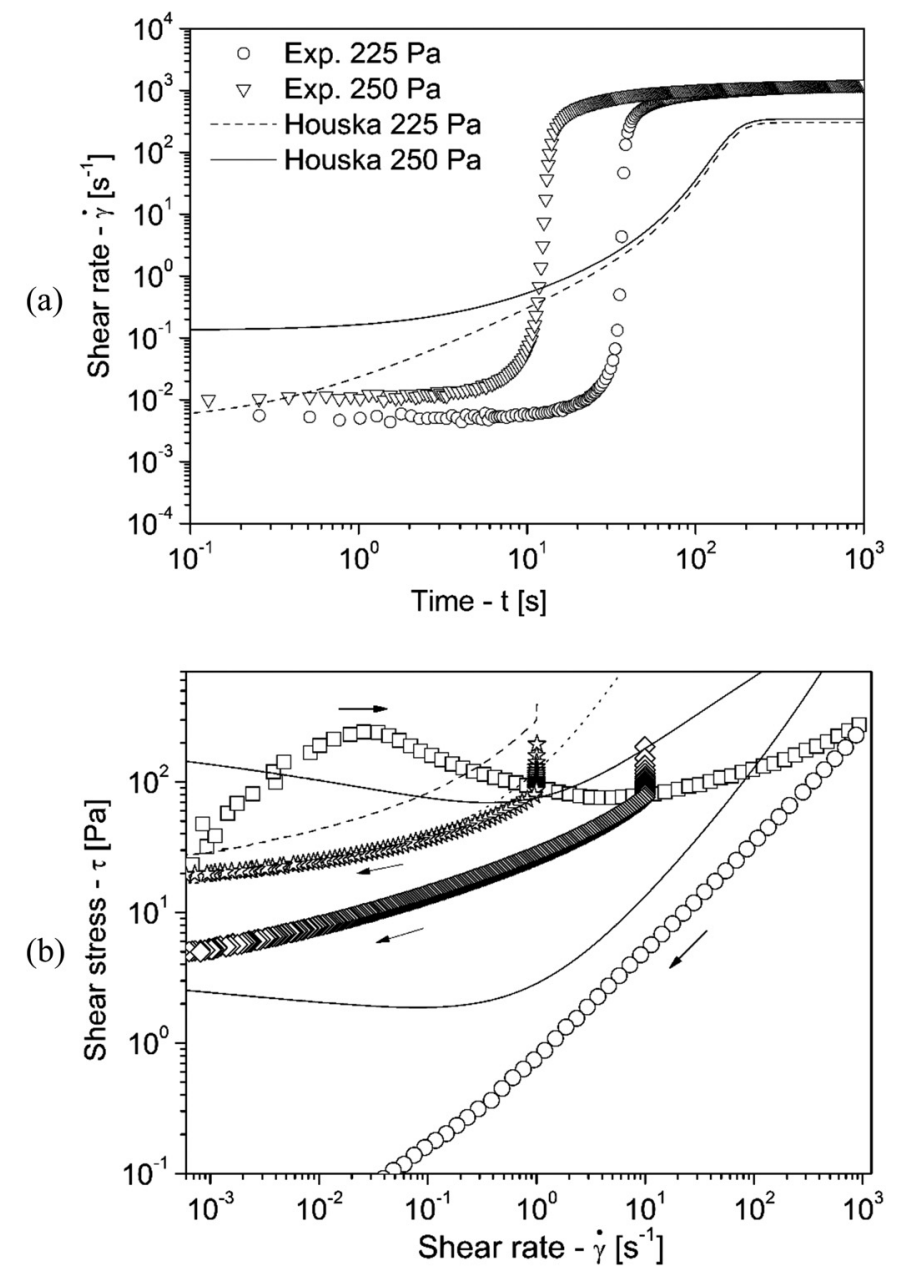

FIG. 14. (a) Houska model (lines) and experimental data (symbols) comparisons for the same imposed shear stress cases as in Fig. 11. (b) Comparisons for the same imposed shear rate cases as in Fig. 12.

temperatures in the range from 4 to $16^{\circ} \mathrm{C}$ are similar, i.e., they may be superimposed by a simple vertical shift. This suggests that the evolution of the structure for these systems is similar. Non-Newtonian effects suddenly disappear at a temperature of $20^{\circ} \mathrm{C}$ : The equilibrium curve is now close to a Newtonian curve (see Fig. 16).

Another series of tests at temperatures higher than the WAT were also carried out according to the following procedure. A sample was charged in the rheometer, heated to $60^{\circ} \mathrm{C}$ and a shear rate ramp up and down cycle from $10^{-4}$ to $100 \mathrm{~s}^{-1}$ in 6 min was performed. Next, the oil was cooled at rest until $50^{\circ} \mathrm{C}$ at $-1{ }^{\circ} \mathrm{C} / \mathrm{min}$, left $5 \mathrm{~min}$ at rest for temperature equilibration and another shear rate ramp cycle was performed. That continued to the temperatures of 30 and $20^{\circ} \mathrm{C}$. The ramps up and down at all temperatures superposed each other, as expected for a simple liquid behavior. These curves have a slope 1 in logarithmic scale, indicating a Newtonian behavior (see Fig. 16). For the temperature of $20^{\circ} \mathrm{C}$, it can be seen in Fig. 16 that the two measurement methods originated curves are almost superimposing. Some difference between them can be seen at low shear rates, where wax crystals, already present in a small quantity at that temperature, may be the cause of the emerging time-dependent effects. 


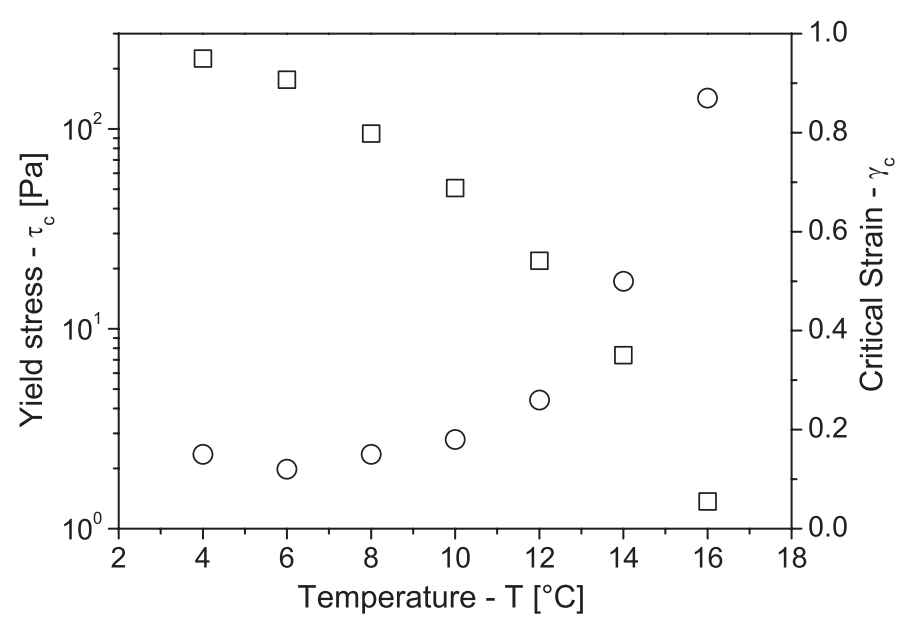

FIG. 15. Yield stress ( $\square$ - squares) and critical deformation ( $\bigcirc$-circles) as a function of temperature determined from tests similar to that of Fig. 3 at shear rate of $5 \times 10^{-3} \mathrm{~s}^{-1}$ for crude oil A.

The HB parameters that fit the curves in Fig. 16 in the form $\tau_{e q}=\tau_{0, e q}(T)$ $+k_{0, e q}(T) \dot{\gamma}^{n(T)}$ are shown in Table III.

Finally, the following model represents the data for the equilibrium curve:

$$
\tau_{e q}=\theta(T)\left[\tau_{0, e q}\left(4^{\circ} \mathrm{C}\right)+k_{0, e q}\left(4^{\circ} \mathrm{C}\right) \dot{\gamma}^{0.5}\right] \quad \text { for } T \leq 20^{\circ} \mathrm{C},
$$

where $\theta(T)=18.4-T / 16$ and

$$
\tau_{e q}=\beta(T) \dot{\gamma} \quad \text { for } T>20^{\circ} \mathrm{C} .
$$

where $\beta(T)=5.8 \times 10^{5} \exp (-T / 18.3)$.

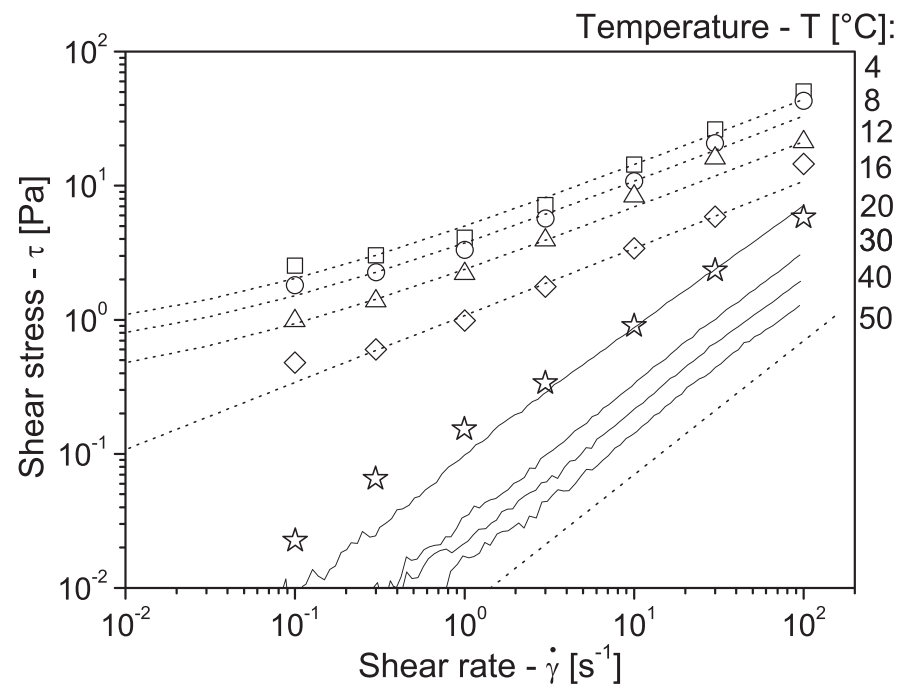

FIG. 16. Equilibrium curves for crude oil A after cooling to different temperatures. Dotted lines correspond to a $\mathrm{HB}$ model fitted to data (see values in Table III). For temperatures higher than $20^{\circ} \mathrm{C}$ (continuous lines), the data were obtained from a sweep test. 
TABLE III. HB parameters of crude oil A fitted to equilibrium curves of Fig. 16.

\begin{tabular}{lccc}
\hline \hline Temperature $\left({ }^{\circ} \mathrm{C}\right)$ & $\tau_{0, e q}(\mathrm{~Pa})$ & $k_{0, e q}\left(\mathrm{~Pa} \mathrm{~s}^{\mathrm{n}}\right)$ & $n$ \\
\hline 4 & 0.66 & 4.33 & 0.5 \\
8 & 0.48 & 3.26 & 0.5 \\
12 & 0.27 & 2.11 & 0.5 \\
16 & 0 & 1.08 & 0.5 \\
20 & 0 & 0.074 & 1 \\
30 & 0 & 0.033 & 1 \\
40 & 0 & 0.021 & 1 \\
50 & 0 & 0.014 & 1 \\
\hline \hline
\end{tabular}

Cooling conditions may be very different from pipe to pipe. For a pipeline exporting the crude oil in the liquid phase under laminar flow, for example, cooling rates are expected to be low. Under that condition, for a waxy oil that is cooling under shear, its apparent viscosity should be always at the equilibrium state at any position inside the pipe that corresponds to a certain temperature. When the oil achieves an equilibrium temperature, its viscosity should remain constant as well, if the shear rate is not changed. That was observed in experiments with a waxy oil cooling under shear by Mendes et al. (2014).

\section{CONCLUSION}

The time-dependent rheological behavior of two waxy crude oils was analyzed. Using creep tests and constant shear rate tests, the main rheological features of those fluids were drawn. Beyond the solid-liquid transition, an irreversible destructuring flow takes place. Transient shear rate tests showed that at low deformations the destructuring flow is related uniquely to the deformation experienced by the fluid. But at high deformations shear rate variations play an important role and the flow history becomes crucial for determining steady state flow. Increases in shear rate beyond its historical maximum value will further reduce the fluid viscosity, imposing an instantaneous and thixotropic response, both with magnitudes depending on how far the fluid is from its equilibrium state for the corresponding shear rate. During the destructuring flow, when the shear rate is decreased, the viscosity augmentation will be smaller if the flow is closer to its equilibrium condition, until achieving a simple Newtonian behavior at the equilibrium. So, for this type of fluid, the equilibrium flow curve depends on the flow history and it is tuned by the maximum shear rate experienced by the fluid. That physical characteristic should be taken into account in rheometrical procedures, as waiting for flow stabilization in stepwise increasing shear rates for matching the history of each layer of the fluid in a pipeline laminar flow. All those features were mathematically modeled as a function of the deformation and shear rate history. The model presented good agreement with experimental data from transient imposed shear rate tests and flow start from an imposed shear stress.

The capacity of taking the flow history into account for determining the fluid rheological behavior at equilibrium state is an important improvement of the proposed model. In practice, the application of this model will also improve the transient flow prediction of a pipeline flow restart with gelled oil. The main features of the model were developed to account for the complex fluid responses experimentally observed. Comparing to the Houska model, for example, predictions of flow rate changes and shut-in during destructuring flows shall be improved as well. 


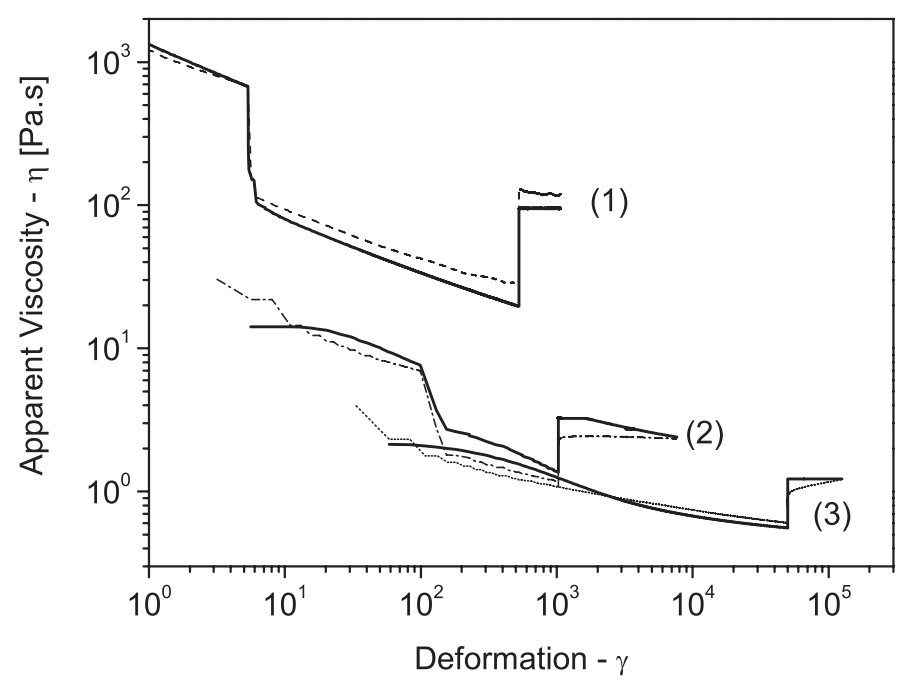

FIG. 17. First group of data comparing simulated (continuous lines) and measured data for three shear rate step changes experiments presented in Fig. 4.

\section{APPENDIX: COMPARISON WITH FITTING DATA}

The destructuring flow data of Fig. 4 used to fit the model parameters are compared to the model predictions for those six experiments in Figs. 17 and 18. The simulated apparent viscosities are represented by the continuous lines. For each case, a sample of crude oil A was cooled at rest from 60 to $4{ }^{\circ} \mathrm{C}$ at $-1{ }^{\circ} \mathrm{C} / \mathrm{min}$ and held at $4{ }^{\circ} \mathrm{C}$ for $20 \mathrm{~min}$ before starting the tests. In Fig. 17, the following tests are presented: (1) Start at $0.1 \mathrm{~s}^{-1}$, change to $1 \mathrm{~s}^{-1}$ at 10 strain units, back to $0.1 \mathrm{~s}^{-1}$ at 500 strain units; (2) start at $10 \mathrm{~s}^{-1}$, change to $100 \mathrm{~s}^{-1}$ at 100 strain units, back to $10 \mathrm{~s}^{-1}$ at 1000 strain units; (3) start at $100 \mathrm{~s}^{-1}$ and change to $10 \mathrm{~s}^{-1}$ at 50000 strain units. Figure 18 shows the comparison for the following procedures: (1) Start at $1 \mathrm{~s}^{-1}$, change to $10 \mathrm{~s}^{-1}$ at 10 strain units, back

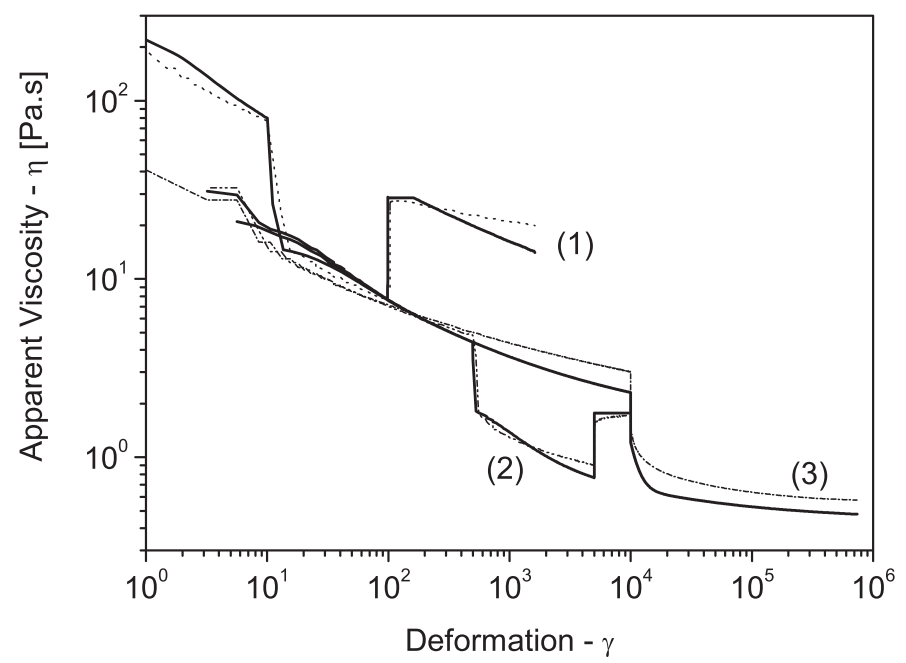

FIG. 18. Second group of data on the comparison between simulated (continuous lines) and measured data for shear rate step changes experiments presented in Fig. 4. 


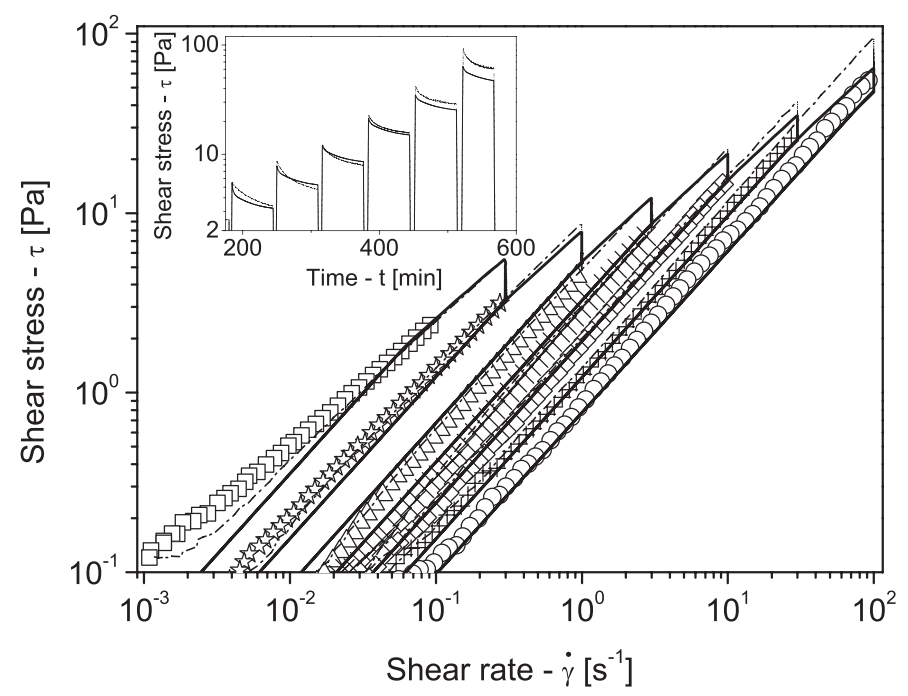

FIG. 19. Comparison between simulated (continuous lines) and measured (symbols and dashed lines) data for the experimental procedure described in Fig. 5(a), starting at the equilibrium condition at $0.1 \mathrm{~s}^{-1}$. The inset presents the equilibration steps at constant shear rate.

to $1 \mathrm{~s}^{-1}$ at 100 strain units; (2) start at $10 \mathrm{~s}^{-1}$, change to $100 \mathrm{~s}^{-1}$ at 1000 strain units, back to $10 \mathrm{~s}^{-1}$ at 5000 strain units; (3) start at $10 \mathrm{~s}^{-1}$ and change to $100 \mathrm{~s}^{-1}$ at 10000 strain units.

According to the model construction features, the global trends observed in those experiments are captured by the model. The parameters used to control the magnitude of the instantaneous viscosity changes in function of instantaneous shear rate changes, $\alpha$ in Eq. (9), and the characteristic time of the destructuring process at constant shear rate, $t_{0}$ in Eq. (10), may still be improved but the order of magnitude of viscosity jumps and long destructuring flows are in a good agreement with the experiments.

Figure 19 presents the comparison between model predictions and the fluid rheological behavior close to the equilibrium. The experiment procedure is that of Fig. 5(a). The simulated data, represented by the continuous line, start at the equilibrium condition at $0.1 \mathrm{~s}^{-1}$ and follow the same imposed shear rate history as in the experiment. The up (dashed lines) and down (symbols) ramps of Fig. 7 are repeated in Fig. 19. The inset shows the measured data (dashed lines) at the equilibration steps (constant shear rate steps) of Fig. 5 in comparison to the simulated data (continuous lines).

The qualitative agreement between measured and simulated data is good. The most important differences are noticed for shear rates below $10^{-2} \mathrm{~s}^{-1}$ and at the destructuring flow of the increasing shear rate ramps from 10 to $30 \mathrm{~s}^{-1}$ and from 30 to $100 \mathrm{~s}^{-1}$. Nevertheless, the general agreement is good for this comparison covering six decades of shear rate variations and about $6.5 \mathrm{~h}$ of flow.

\section{References}

Cawkwell, M. G., and M. E. Charles, "Characterization of Canadian artic thixotropic gelled crude oils utilizing an eight-parameter model," J. Pipelines 7, 251-264 (1989).

Chang, C., D. V. Boger, and Q. D. Nguyen, "The yielding of waxy crude oils," Ind. Eng. Chem. Res. 37, 1551-1559 (1998).

Chang, C., Q. D. Nguyen, and H. P. Rønningsen, "Isothermal start-up of pipeline transporting waxy crude oil," J. Non-Newtonian Fluid Mech. 87, 127-154 (1999). 
Coussot, P., Rheometry of pastes, suspensions, and granular materials: Applications in industry and environment (John Wiley \& Sons, Inc., Hoboken, NJ, 2005).

Coussot, P., A. I. Leonov, and J. M. Piau, "Rheology of concentrated dispersed systems in low molecular weight matrix," J. Non-Newtonian Fluid Mech. 46, 179-217 (1993).

Coussot, P., Q. D. Nguyen, H. T. Huynh, and D. Bonn, "Viscosity bifurcation in thixotropic, yielding fluids," J. Rheol. 46(3), 573-589 (2002).

de Souza Mendes, P. R., “Thixotropic elasto-viscoplastic model for structured fluids,” Soft Matter 7, 2471-2483 (2011).

Dimitriou, C. J., and G. H. McKinley, "A comprehensive constitutive law for waxy crude oil: A thixotropic yield stress fluid," Soft Matter 10, 6619-6644 (2014).

Ding, J., J. Zhang, H. Li, F. Zhang, and X. Yang, "Flow behavior of Daqing waxy crude oil under simulated pipelining conditions," Energy Fuels 20, 2531-2536 (2006).

Dullaert, K., and J. Mewis, "A structural kinetics model for thixotropy," J. Non-Newtonian Fluid Mech. 139, 21-30 (2006).

Ekweribe, C., F. Civan, H. S. Lee, and P. Singh, "Effect of system pressure on restart conditions of subsea pipelines," in SPE Annual Technical Conference and Exhibition (2008), Vol. 3, pp. 1754-1775, SPE 115672.

El-Gendy, H., M. Alcoutlabi, M. Jemmett, M. Deo, J. Magda, R. Venkatesan, and A. Montesi, "The propagation of pressure in a gelled waxy oil pipeline as studied by particle imaging velocimetry," AIChE 58, 302-311 (2012).

Hénaut, I., and F. Brucy, "Description rhéologique des bruts paraffiniques gélifiés," Congrés du Groupe Français de Rhéologie, 2001.

Hénaut, I., O. Vincké, and F. Brucy, "Waxy crude oil restart: Mechanical properties of gelled oils," in SPE Annual Technical Conference and Exhibition (1999), SPE 56771.

Houska, M., "Engineering aspects of the rheology of thixotropic liquids," Ph.D. thesis, Faculty of Mechanical Engineering, Czech Technical University of Prague-CVUT, 1981.

Jia, B., and J. Zhang, "Yield behavior of waxy crude gel: Effect of isothermal structure development before prior applied stress,” Ind. Eng. Chem. Res. 51, 10977-10982 (2012).

Kané, M., M. Djabourov, and J. L. Volle, "Rheology and structure of waxy crude oils in quiescent and under shearing conditions," Fuel 83, 1591-1605 (2004).

Kané, M., M. Djabourov, J. L. Volle, J. P. Lechaire, and G. Frebourg, "Morphology of paraffin crystals in waxy crude oils cooled in quiescent conditions and under flow," Fuel 82, 127-135 (2003).

Lin, M., C. Li, F. Yang, and Y. Ma, "Isothermal structure development of Qinghai waxy crude oil after static and dynamic cooling,” J. Pet. Sci. Technol. 77, 351-358 (2011).

Lopes-da-Silva, J. A., and J. A. P. Coutinho, "Analysis of the isothermal structure development in waxy crude oils under quiescent conditions," Energy Fuels 21, 3612-3617 (2007).

Magda, J. J., H. El-Gendy, K. Oh, M. D. Deo, A. Montesi, and R. Venkatesan, "Time-dependent rheology of a model waxy crude oil with relevance to gelled pipeline restart," Energy Fuels 23, 1311-1315 (2009).

Marchesini, F. H., A. A. Alicke, P. R. de Souza Mendes, and C. M. Ziglio, "Rheological characterization of waxy crude oils: Sample preparation,” Energy Fuels 26(5), 2566-2577 (2012).

Mendes, R., G. Vinay, G. Ovarlez, and P. Coussot, "Reversible and irreversible destructuring flow in waxy oils: An MRI study," J. Non-Newtonian Fluid Mech. (published online).

Mewis, J., and N. J. Wagner, “Thixotropy,” Adv. Colloid. Interface Sci. 147-148, 214-227 (2009).

Møller, P. C. F., S. Rodts, M. A. J. Michel, and D. Bonn, "Shear banding and yield stress in soft glassy materials," Phys. Rev. E 77, 041507 (2008).

Oh, K., M. Jemmett, and M. Deo, "Yield behavior of gelled waxy oil: Effect of stress application in creep ranges,” Ind. Eng. Chem. Res. 48, 8950-8953 (2009).

Ovarlez, G., S. Rodts, X. Chateau, and P. Coussot, "Phenomenology and physical origin of shear localization and shear banding in complex fluids," Rheol. Acta 48, 831-844 (2009).

Philips, D. A., I. N. Forsdyke, I. R. McCracken, and P. D. Ravenscroft, "Novel approaches to waxy crude restart: Part 1: Thermal shrinkage of waxy crude oil and the impact for pipeline restart,” J. Pet. Sci. Technol. 77, 237-253 (2011a).

Philips, D. A., I. N. Forsdyke, I. R. McCracken, and P. D. Ravenscroft, "Novel approaches to waxy crude restart: Part 2: An investigation of flow events following shut down,” J. Pet. Sci. Technol. 77, 286-304 (2011b). 
Pignon, F., A. Magnin, and J. Piau, "Thixotropic colloidal suspensions and flow curves with minimum: Identification of flow regimes and rheometric consequences," J. Rheol. 40, 573-587 (1996).

Rønningsen, H. P., "Rheological behaviour of gelled, waxy North Sea crude oils,” J. Pet. Sci. Eng. 7, 177-213 (1992).

Rønningsen, H. P., B. Bjorndal, A. B. Hansen, and W. B. Pedersen, "Wax precipitation from north sea crude oils. 1. Crystallization and dissolution temperatures, and Newtonian and non-Newtonian flow properties," Energy Fuels 5, 895-908 (1991).

Sestak, J., M. E. Charles, M. G. Cawkwell, and M. Houska, "Start-up of gelled crude oil pipelines," J. Pipelines 6, 15-24 (1987).

Tanner, R. I., Engineering Rheology (Oxford University, Oxford, 2000).

Teng, H., and J. Zhang, "Modeling the thixotropic behavior of waxy crude," Ind. Eng. Chem. Res. 52, 8079-8089 (2013).

Venkatesan, R., N. R. Nagarajan, K. Paso, Y. B. Yi, A. M. Sastry, and H. S. Fogler, "The strength of paraffin gels formed under static and flow conditions," Chem. Eng. Sci. 60, 3587-3598 (2005).

Visintin, R. F. G., R. Lapasin, E. Vignati, P. D’Antona, and T. P. Lockhart, "Rheological behavior and structural interpretation of waxy crude oil gels," Langmuir 21, 6240-6249 (2005).

Wachs, A., G. Vinay, and I. Frigaard, "A 1.5D numerical model for the start up of weakly compressible flow of a viscoplastic and thixotropic fluid in pipelines," J. Non-Newtonian Fluid Mech. 159, 81-94 (2009).

Wang, Y., and Q. Huang, "Evaluation of measurement methods of waxy crude oil thixotropy," J. Dispersion Sci. Technol. 35(9), 1255-1263 (2014).

Zhao, Y., L. Kumar, K. Paso, H. Ali, J. Safieva, and J. Sjöblom, "Gelation and breakage of model wax-oil systems: Rheological properties and model development,” Ind. Eng. Chem. Res. 51, 8123-8133 (2012). 